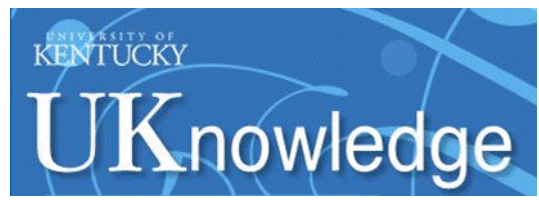

University of Kentucky

UKnowledge

Lafora Epilepsy Cure Initiative Faculty

Publications

Lafora Epilepsy Cure Initiative

$4-14-2020$

\title{
Polyglucosan Body Structure in Lafora Disease
}

\author{
M. Kathryn Brewer \\ University of Kentucky, brewermk@gmail.com \\ Jean-Luc Putaux \\ University of Grenoble Alpes, France \\ Alberto Rondon \\ University of Kentucky, alro272@uky.edu \\ Annette M. Uittenbogaard \\ University of Kentucky, auitt0@uky.edu \\ Mitchell A. Sullivan \\ The University of Queensland, Australia
}

See next page for additional authors

Follow this and additional works at: https://uknowledge.uky.edu/leci_facpub

Part of the Biochemistry Commons, and the Molecular and Cellular Neuroscience Commons

Right click to open a feedback form in a new tab to let us know how this document benefits you.

\section{Repository Citation}

Brewer, M. Kathryn; Putaux, Jean-Luc; Rondon, Alberto; Uittenbogaard, Annette M.; Sullivan, Mitchell A.; and Gentry, Matthew S., "Polyglucosan Body Structure in Lafora Disease" (2020). Lafora Epilepsy Cure Initiative Faculty Publications. 1.

https://uknowledge.uky.edu/leci_facpub/1

This Article is brought to you for free and open access by the Lafora Epilepsy Cure Initiative at UKnowledge. It has been accepted for inclusion in Lafora Epilepsy Cure Initiative Faculty Publications by an authorized administrator of UKnowledge. For more information, please contact UKnowledge@lsv.uky.edu. 


\section{Polyglucosan Body Structure in Lafora Disease}

Digital Object Identifier (DOI)

https://doi.org/10.1016/j.carbpol.2020.116260

\section{Notes/Citation Information}

Published in Carbohydrate Polymers.

() 2020 Elsevier Ltd.

(๑) 2020. This manuscript version is made available under the CC-BY-NC-ND 4.0 license

http://creativecommons.org/licenses/by-nc-nd/4.0/.

The document available for download is the authors' post-peer-review final draft of the article.

\section{Authors}

M. Kathryn Brewer, Jean-Luc Putaux, Alberto Rondon, Annette M. Uittenbogaard, Mitchell A. Sullivan, and Matthew S. Gentry 
1 Title: Polyglucosan body structure in Lafora disease

2

3 Keywords (up to 10): glycogen; starch; epilepsy; polyglucosan bodies; Lafora bodies;

4 Lafora disease; glycogen storage disease

5

6 Authors: M. Kathryn Brewer1,2,3, Jean-Luc Putaux4, Alberto Rondon1, Annette

7 Uittenbogaard1, Mitchell A. Sullivan5, Matthew S. Gentry1,2.

8 Affiliations:

9 1 Department of Molecular and Cellular Biochemistry, University of Kentucky College of 10 Medicine, Lexington, KY 40536, USA.

112 Lafora Epilepsy Cure Initiative, Epilepsy and Brain Metabolism Center, and Center for

12 Structural Biology, University of Kentucky College of Medicine, Lexington, KY 40536,

13 USA.

143 Institute for Research in Biomedicine (IRB Barcelona), 08028 Barcelona, Spain.

154 Univ. Grenoble Alpes, CNRS, CERMAV, F-38000 Grenoble, France.

165 Glycation and Diabetes Group, Mater Research Institute-The University of Queensland,

17 Translational Research Institute, Woolloongabba, Queensland, Australia.

18

19 Correspondence: Matthew S. Gentry, Department of Molecular and Cellular Biochemistry,

20 University of Kentucky College of Medicine, Lexington, KY 40536, USA.

21 Phone: +1 859323 8482; Fax: +1 859323 5505; Email: matthew.gentry@uky.edu 


\section{Abbreviations}

27 PGB: polyglucosan body; LD: Lafora disease; LB: Lafora body; GSD: glycogen storage

28 disease; CLD: chain length distribution; TEM: transmission electron microscopy; WAXS:

29 wide-angle X-ray scattering; SAXS: small-angle X-ray scattering; DIC: differential

30 interference contrast; SmLBs: skeletal muscle LBs; BrLBs: brain LBs; HtLBs: heart LBs;

31 PAPS: potato amylopectin starch; SEC: size exclusion chromatography; $R \mathrm{~h}$ : hydrodynamic

32 radius; WT: wild-type.

33 


\section{Abstract (150 words or less)}

35 Abnormal carbohydrate structures known as polyglucosan bodies (PGBs) are associated with

36 neurodegenerative disorders, glycogen storage diseases (GSDs), and aging. A hallmark of the

37 GSD Lafora disease (LD), a fatal childhood epilepsy caused by recessive mutations in the

$38 E P M 2 A$ or EPM2B genes, are cytoplasmic PGBs known as Lafora bodies (LBs). LBs result

39 from aberrant glycogen metabolism and drive disease progression. They are abundant in

40 brain, muscle and heart of LD patients and Epm2a-/ and Epm2b-/ mice. LBs and PGBs are

41 histologically reminiscent of starch, semicrystalline carbohydrates synthesized for glucose

42 storage in plants. In this study, we define LB architecture, tissue-specific differences, and

43 dynamics. We propose a model for how small polyglucosans aggregate to form LBs. LBs are

44 very similar to PGBs of aging and other neurological disorders, and so these studies have

45 direct relevance to the general understanding of PGB structure and formation. 


\section{Introduction}

Polyglucosan bodies (PGBs) are a common feature of glycogen storage diseases (GSDs), neurodegenerative diseases, and physiological aging (Cavanagh, 1999; Duran \& Guinovart, 2015; Rohn, 2015). PGBs range in size from 2 to $50 \mu \mathrm{m}$ in diameter and, unlike the proteinaceous inclusion bodies of neurodegenerative diseases, they are primarily made of an abberant glucose polymer called polyglucosan (Raben et al., 2001). These glucose polymers are considered "abberant" because they differ significantly in structure and appearance from the normal glucose polymers of mammalian tissues, namely glycogen. Subtle chemical and ultrastructural differences distinguish the PGBs of various pathologies (Cavanagh, 1999).

What unites them is their chemical resemblance to mammalian glycogen and plant starch, the two major forms of glucose storage in living organisms (Emanuelle, Brewer, Meekins, \& Gentry, 2016).

Glycogen is a polysaccharide comprised of $\alpha-1,4-$ linked linear chains of glucose with $\alpha$ 1,6-linked branches. Starch contains two types of polysaccharides, amylopectin and amylose.

61 Amylopectin is the major component of starch and, like glycogen, contains both $\alpha-1,4$ and $\alpha$ -

62 1,6-linkages. Amylose, the minor constituent of starch (typically 15-30\% of total starch weight), is comprised almost exclusively of linear $\alpha-1,4$-linked chains with few branch points. Although glycogen and amylopectin have identical glycosidic bonding, differences in chain length and branching frequency give them distinct properties. The linear chains of glycogen have approximately 13 glucose units and two branch points, making glycogen a water-soluble, continuously branched macromolecule that is designed for rapid glucose release (Melendez-Hevia, Waddell, \& Shelton, 1993; Roach, Depaoli-Roach, Hurley, \& Tagliabracci, 2012). In contrast, the linear glucose chains of amylopectin contain on average 20-30 glucose units and branch points are clustered, although the arrangement of the clusters is still under investigation (Bertoft, 2017; Jane et al., 1999). The long, linear regions of the 
glucan chains intertwine to form double helices, producing crystalline layers (called lamellae) interleaved with more hydrated, amorphous regions containing the branch points. The crystalline and amorphous lamellae radiate from a central origin, and as a result, starch granules are a densely packed, semicrystalline glucose cache that can reach up to $100 \mu \mathrm{m}$ in diameter in some plant tissues (Emanuelle et al., 2016; Lourdin et al., 2015). Amylose is interspersed among the amylopectin chains (Bertoft, 2017). Starch and glycogen both contain covalently bound phosphate, located at the 3- and 6-hydroxlys in amylopectin and 2-, 3-, and 6-hydroxyls in glycogen (DePaoli-Roach et al., 2014; Nitschke et al., 2013; Ritte et al., 2006; Young et al., 2019). Amylopectin contains higher levels of phosphate than glycogen: 0.1$0.5 \%$ versus $0.064-0.25 \%$ by weight, depending on the plant or tissue source (Gentry, Dixon, \& Worby, 2009).

Starch-like structures in the brains of elderly patients were first described in 1836 by J. E. Purkinje, who named them corpora amylacea (Latin for "starch-like bodies"). In 1854, Rudolf Virchow observed a substance in the diseased nervous system that stained with iodine in a manner similar to plant starch, coining the term "amyloid" (Virchow, 1854).

Subsequently, others also described "amyloid" deposits that were later discovered to be proteinaceous, so the term now typically refers to the inclusions of Alzheimer's and other amyloidoses (Kyle, 2001; Sipe \& Cohen, 2000). But corpora amylacea and PGBs are, in fact, true amyloid: in addition to their similarity in size and shape and staining characteristics to starch, they are comprised primarily of insoluble glucose polymers with chain lengths longer than normal glycogen (Cafferty et al., 1991; Cavanagh, 1999; Sakai, Austin, Witmer, \& Trueb, 1969). In some cases, these PGBs also contain elevated phosphate (Sullivan et al., 2019). Since glycogen synthase is the only known enzyme able to catalyze glucose polymerization in vivo in mammals, PGBs are considered a pathological result of aberrant glycogen metabolism (Duran \& Guinovart, 2015; Raben et al., 2001; Roach et al., 2012). 

that manifests during the teen years and leads to early death (Akman, Oldfors, \& DiMauro, 2015; Gentry et al., 2020; Gentry, Guinovart, Minassian, Roach, \& Serratosa, 2018; Minassian, 2001). LD is characterized by cytoplasmic PGBs known as Lafora bodies (LBs) that are found in neurons, astrocytes, skeletal and cardiac myocytes, and other cell types 102 (Augé, Pelegrí, et al., 2018; Criado et al., 2012; Rubio-Villena et al., 2018; Van Heycop Ten Ham, 1975). Like other PGBs, LBs from human tissues were described as starch-like in the early- and mid-1900s based on their chemical and structural characteristics (Lafora, 1911; Yokoi, Austin, Witmer, \& Sakai, 1968). Recessive mutations in either of the Epilepsy, progressive myoclonus 2 genes (EPM2A or EPM $2 B$ ) cause LD in humans, and Epm2a-/ and

107 Epm $2 b$ - - mice recapitulate the disease with LB accumulation, neurodegeneration, and seizures (Criado et al., 2012; DePaoli-Roach et al., 2010; Ganesh et al., 2002; Tiberia et al., 2012; Valles-Ortega et al., 2011). EPM2A encodes laforin, the mammalian glycogen

110 phosphatase, and EPM2B encodes malin, an E3 ubiquitin ligase (Gentry et al., 2007; Gentry, 111 Worby, \& Dixon, 2005; Worby, Gentry, \& Dixon, 2006). The three-dimensional structure of 112 laforin has been determined, elucidating the mechanism of its glycogen phosphatase activity 113 (Raththagala et al., 2015). Both laforin and malin are believed to regulate the architecture of 114 glycogen molecules and prevent the formation of LBs, although this mechanism has not yet 115 been elucidated (Sullivan, Nitschke, Steup, Minassian, \& Nitschke, 2017). Multiple groups 116 have established that LBs drive neurodegeneration and epilepsy using these LD mouse 117 models and complementary fly models (reviewed by (Gentry et al., 2018).

118 The polysaccharides from Epm2a-/ and Epm2b-/ mice that have been characterized were 119 purified based on a method first described in 1909 by Pflüger for purifying glycogen, in 120 which the tissue is boiled in $\mathrm{KOH}$ and the polysaccharides are precipitated with ethanol 121 (Good, 1933; Pflüger, 1909). This method does not separate glycogen from the LBs. 
122 Additionally, the Pflüger-purified polysaccharides differ in morphology from LBs observed

123 via microscopy in LD tissue sections. When analyzed by transmission electron microscopy

124 (TEM) the Pflüger-purified particles appear more aggregated than wild-type glycogen

125 (Tagliabracci et al., 2008), but they are dramatically smaller (15-65 $\mathrm{nm}$ ) than the micron-

126 sized LBs observed in tissue sections (Minassian, 2001). Purification of LBs by the Pflüger

127 method likely disrupts LB morphology and also does not separate glycogen from the LB.

128 More recently, the "soluble" and "insoluble" fractions of glycogen were separated after tissue

129 homogenization and analyzed individually, revealing that only the "insoluble" fraction had an

130 altered chain length (Sullivan et al., 2019). The "insoluble" fraction is likely to correspond to

131 the LBs and the "soluble" fraction to normal glycogen, but this was not clearly demonstrated.

132 It is conceivable that LBs are aggregates of polyglucosan (i.e. abnormal polysaccharide)

133 molecules, and that the Pflüger method breaks apart the LBs and intermixes the polyglucosan

134 molecules comprising LBs with normal, soluble glycogen particles. In the case of starch,

135 many studies have shown that the application of heat and moisture results in solubilization

136 and irreversible disruption of the starch granular structure (Ratnayake \& Jackson, 2009).

137 A significant body of work has been performed to define the chemical, physical and

138 structural properties of different types of starch, which vary depending on plant species and

139 tissue type (reviewed by (Lourdin et al., 2015). One of the major features that distinguishes

140 starch from glycogen is its semicrystalline, lamellar organization that can be detected with

141 diffraction techniques such as wide- and small-angle X-ray scattering (WAXS and SAXS,

142 respectively). Treatment of starch granules with mild hydrochloric acid, a process known as

143 lintnerization, preferentially hydrolyzes the amorphous regions, leaving crystalline regions

144 intact and providing valuable information about granule architecture (Gerard, Planchot,

145 Colonna, \& Bertoft, 2000; Srichuwong, Isono, Mishima, \& Hisamatsu, 2005; Wikman, 
146 Blennow, \& Bertoft, 2013). Despite the similarities between PGBs and starch, comparable

147 methods have not yet been applied to investigate the structure of native PGBs.

148 In prior studies, variations of the Pflüger method were used to purify LD polyglucosan for

149 biochemical analysis. NMR and enzymatic analyses demonstrated that LD polyglucosan

150 contains elevated phosphate linked to the C2-, C3- and C6- hydroxyls of the glucose moieties

151 in relatively equivalent ratios (DePaoli-Roach et al., 2015; Nitschke et al., 2013; Tagliabracci

152 et al., 2011). Chain length distribution analysis showed an increase in longer chains in

153 polyglucosan from muscle and brain, which causes the polyglucosan to precipitate (Irimia et

154 al., 2015; Nitschke et al., 2017; Sullivan et al., 2019). These samples were always denatured;

155 and native LBs were not analyzed. Furthermore, no biochemical study of isolated

156 polyglucosan from cardiac tissue was performed. We recently described a novel method for

157 isolating native LBs from Epm $2 a$-/ and Epm2b-/- mice using a protocol that does not rely on

158 the Pflüger method, separates LBs from glycogen, and preserves the endogenous size and

159 structure of the LBs (Brewer et al., 2019). Using light microscopy and scanning electron

160 microscopy, we showed that native LBs from brain, heart, and skeletal muscle have distinct

161 sizes and morphologies, typically ranging from 2 to $10 \mu \mathrm{m}$ in diameter, consistent with their

162 distinct appearances in fixed tissue sections. We hypothesized that LBs possess starch-like

163 biophysical and chemical properties, beyond just histochemical similarities, that distinguish

164 them from normal glycogen. In the present study, we define the physiochemical and

165 structural characteristics of native LBs isolated from different tissues. Combining these

166 methods with thermal, mechanical and chemical treatments to disassemble LBs allows for a

167 model of how polyglucosan molecules aggregate to form LBs in LD. Although not all PGBs

168 are identical, the corpora amylacea of aging and neurodegeneration and the PGBs of multiple

169 GSDs bear striking similarities to LBs. We propose that PGBs may represent a common 
pathological phenomenon resulting from misregulated glycogen metabolism, which has particularly detrimental consequences in the brain.

172

\section{Methods and Materials}

2.1. Purification of native LBs from Epm2a-- mice

All animal handling and procedures were approved by the University of Kentucky Institutional Animal Care and Use Committee (IACUC). Epm2a-/ mice have been previously described (DePaoli-Roach et al., 2012; Ganesh et al., 2002). Native LBs from 19-24 month old Epm2a-^- mice were purified as previously described (Brewer et al., 2019).

\subsection{Confocal microscopy} confocal microscopy, LBs or corn starch (Sigma) were stained with 20× Lugol's iodine, embedded in Mowiol 4-88 (Sigma cat \#81381) on glass slides, and visualized using the TRITC channel (561 nm excitation laser) and differential interference contrast (DIC) using a Nikon AR+ Scope. Z-stack images of LBs were captured in $0.1 \mu \mathrm{m}$ steps, starch granules in $0.25 \mu \mathrm{m}$ steps, and 3D deconvolution was performed using the Nikon Elements Advanced

187 Research Software. The Richardson-Lucy deconvolution algorithm was utilized with 10 188 iterations per stack.

\subsection{Small-and wide-angle X-ray scattering (SAXS and WAXS)}

Purified SmLBs and BrLBs were centrifuged at 14,000 rpm, and the wet pellets were poured into $1 \mathrm{~mm}$ (outer diameter) glass capillaries. For comparison purpose, capillaries

193 containing potato amylopectin starch (PAPS) granules (a gift from E. Bertoft, Turku

194 University) and rabbit liver glycogen (Fluka) were prepared as well. All specimens were 
equilibrated for 5 days in a closed chamber maintaining a 93\% relative humidity. The capillaries were flamed-sealed and $\mathrm{X}$-rayed in air by a $\mathrm{Ni}$-filtered $\mathrm{CuK} \alpha$ radiation $(\lambda=0.1542$ nm) using a Philips PW3830 generator operating at $30 \mathrm{kV}$ and $20 \mathrm{~mA}$. Two-dimensional scattering diagrams were recorded on Fujifilm imaging plates placed at a distance of about 5 $\mathrm{cm}$ from the capillary and read offline with a Fujifilm BAS 1800-II bioanalyzer. Diffraction profiles were calculated by rotationally averaging the $2 \mathrm{D}$ diffraction patterns after subtraction of the scattering signal from air. Capillaries containing SmLBs and BrLBs were also heated at $95{ }^{\circ} \mathrm{C}$ and analyzed immediately after heating and after 12 and $36 \mathrm{~h}$ at $4{ }^{\circ} \mathrm{C}$. Considering the small amount of material, the specimens of acid-hydrolyzed LBs were centrifuged inside the capillaries and X-rayed in excess water. SAXS patterns were recorded from wet specimens (i.e. kept in excess water), with the imaging plates placed at a distance of $30 \mathrm{~cm}$ from the capillaries, and scattering profiles were calculated using the same rotational averaging procedure. SAXS data is usually presented as a function of the scattering vector, $q$ (Blazek \& Gilbert, 2011). WAXS profiles are presented as a function of the diffraction angle $(2 \theta)$. The scattering vector $(q)$ is geometrically related to the wavelength of the incident

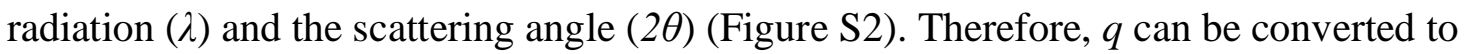

211 distance of the repeating unit $(d)$ in real space using Bragg's law:

$$
q=\frac{4 \pi}{\lambda} \sin \theta=\frac{2 \pi}{d} \quad \text { Equation (1) }
$$

\subsection{LB treatments and light microscopy}

SmLBs and BrLBs were aliquoted (100 $\mu \mathrm{g}$ per tube) and washed in water by centrifugation at $16,000 \mathrm{~g}$ for $1 \mathrm{~min}$. LB pellets were resuspended in $100 \mu \mathrm{L}$ water or $100 \mu \mathrm{L}$ $30 \% \mathrm{KOH}$ for a final concentration of $1 \mathrm{mg} / \mathrm{ml}$. For sonication treatment, five pulses of 5 seconds each were applied at $20 \mathrm{kHz}$ with $25 \%$ amplitude, with one minute on ice between each pulse to prevent sample heating. For heat treatments at $95{ }^{\circ} \mathrm{C}$, samples were heated for 
$22030 \mathrm{~min}$ or $2 \mathrm{~h}$ as indicated. After treatment, $5 \mu \mathrm{L}$ of samples were stained with $2 \mu \mathrm{L} 20 \times$

221 Lugol's iodine, mounted on glass slides with a glass coverslip, and visualized using a Nikon

222 Eclipse E600 using DIC/Nomarski contrast and an AxioCam MRm or Zeiss 512 camera at

223 100x. LBs had a tendency to flocculate, so after staining, samples were carefully triturated

224 with a pipet, added to glass slides, and the coverslip was laid on top. The coverslip was very

225 gently prodded and pressed to disperse the LB clumps and reduce the number of focal planes.

226 Image thresholding was performed using the ImageJ software (National Institutes of Health,

227 USA).

228

2.5. Hydrothermal and lintnerization treatments for transmission electron microscopy (TEM)

230 Two different treatments were performed to disrupt the LB structure and the resulting specimens were observed by TEM. In the first one, dilute aqueous LB suspensions were heated at $95{ }^{\circ} \mathrm{C}$ for $30 \mathrm{~min}$, and TEM specimens were prepared after cooling down at room temperature and after keeping the suspensions for 3 days at $4{ }^{\circ} \mathrm{C}$. The second treatment was carried out by disrupting LBs in $2.2 \mathrm{~N} \mathrm{HCl}$ and incubating the suspensions at $36^{\circ} \mathrm{C}$. This gradual hydrolysis of glucose polymers in mild acid, a process known as lintnerization, is used to study crystalline structure (Bertoft, 2017; Jacobs, Eerlingen, Rouseu, Colonna, \& Delcour, 1998). After 5 days, the suspensions were centrifuged; the pellets were repeatedly washed to neutrality by centrifugation in water and finally re-dispersed in water. Droplets of the various dilute suspensions were deposited onto glow-discharged carbon-coated copper

240 grids. After blotting of the excess liquid, the preparations were negatively stained with 2 wt $\%$ uranyl acetate, allowed to dry and observed with a JEOL JEM 2100-Plus microscope operating at $200 \mathrm{kV}$ and equipped with a Gatan Rio 16 camera. 
Polyglucosan bodies were extracted/dissociated by boiling in $30 \% \mathrm{KOH}$ for $1 \mathrm{~h}$,

followed by ethanol precipitation. This involved adding 4 volumes of ethanol with $15 \mathrm{mM}$

$\mathrm{LiCl}$ and storing at $-30{ }^{\circ} \mathrm{C}$ for $1 \mathrm{~h}$. Samples were then centrifuged at $16,000 \mathrm{~g}$ for $20 \mathrm{~min}$ at 4

${ }^{\circ} \mathrm{C}$. Pellets were resuspended in $200 \mu \mathrm{L}$ of milli-Q water and the ethanol precipitation step was repeated for a total of 3 times. Samples were freeze-dried overnight and then dissolved directly in SEC eluent (50 mM NH4NO3 with $0.02 \%$ sodium azide) at $80{ }^{\circ} \mathrm{C}$ and $350 \mathrm{rpm}$ overnight. Samples were then analyzed using SEC as previously described (Sullivan et al., 2019). Briefly, dissolved polyglucosan ( 2.5 mg.mL-1) was injected into SHIMADZU LC20AD system coupled with differential refractive index detector (Wyatt Technology, USA) using the following columns: SUPREMA preColumn, 1000 and 10,000 (PSS). The column oven was set at $80{ }^{\circ} \mathrm{C}$, and the flow rate was set at $0.3 \mathrm{~mL}$.min-1. The differential refractive index detector was used to obtain the weight distributions, as previously reported (Sullivan et al., 2010). A universal calibration curve was constructed using pullulan standards (ranging from 108 to $1.22 \times 106 \mathrm{Da}$ ), also directly dissolved into the SEC eluent. Using the MarkHouwink relationship the elution volumes were converted to hydrodynamic radius, as previously described in detail (Sullivan et al., 2010). Rat muscle glycogen was used as a reference for normal glycogen particles and was extracted using the same $\mathrm{KOH}$ method, as

262 previously described (Sullivan et al., 2010).

\section{Results}

\subsection{Confocal micrographs of iodine-stained LBs}

Lugol's iodine is a chemical stain that has been used for over a century to detect and differentiate polysaccharides such as glycogen, amylose and amylopectin. Amylose, composed almost entirely of $\alpha-1,4$ linear chains, produces a deep blue-black color when stained with Lugol's iodine, with a maximum absorption at $650 \mathrm{~nm}$ (Rundle, Foster, \& 
270 Baldwin, 1944; Swanson, 1948). The linear glucan chains of amylose form a complex with

271 iodine in the form of a single chromophoric helix. In contrast, the branch points within

272 amylopectin interfere with the interaction, producing a less stable complex with triiodide.

273 The result is a reddish-purple color and a maximum absorption at $550 \mathrm{~nm}$ (Swanson, 1948).

274 Glycogen contains even shorter chains and more frequent branching than amylopectin, and 275 therefore stains poorly with Lugol's iodine. Glycogen yields an absorbance maximum at 440

$276 \mathrm{~nm}$, although this varies slightly depending on glycogen source, i.e. glycogen from different

277 tissues and/or organisms (Archibald et al., 1961).

278 Iodine staining of LBs, corpora amylacea, and other PGBs indicates a branching degree 279 that is more similar to amylopectin than glycogen (Herrick, Twiss, Vladutiu, Glasscock, \& 280 Horoupian, 1994; Reed Jr, Dixon, Neustein, Donnell, \& Landing, 1968; Sakai et al., 1969).

281 We previously showed that native LBs isolated from skeletal muscle, heart tissue, and brain 282 of Epm2a-/ mice stained intensely reddish-brown in Lugol's iodine with an absorbance 283 maximum at approximately 510-520 nm (Brewer et al., 2019). The iodine-stained LBs not 284 only absorb at this wavelength, but they also produce red fluorescence when excited by a 561 $285 \mathrm{~nm}$ confocal laser. Confocal microscopy has previously been used as a sensitive method for visualizing starch granules in vitro and in planta (Bahaji et al., 2011; Ovecka et al., 2012). Zstack images of iodine-stained starch granules visualized using the $561 \mathrm{~nm}$ laser and differential interference contrast (DIC) at high magnification show that only the smooth, outer edge of the granule is stained (Figure 1A and B, Figure S1A through D). This staining

290 pattern indicates that iodine more efficiently penetrates the outer portion than the densely 291 packed semicrystalline interior of the starch granule. 

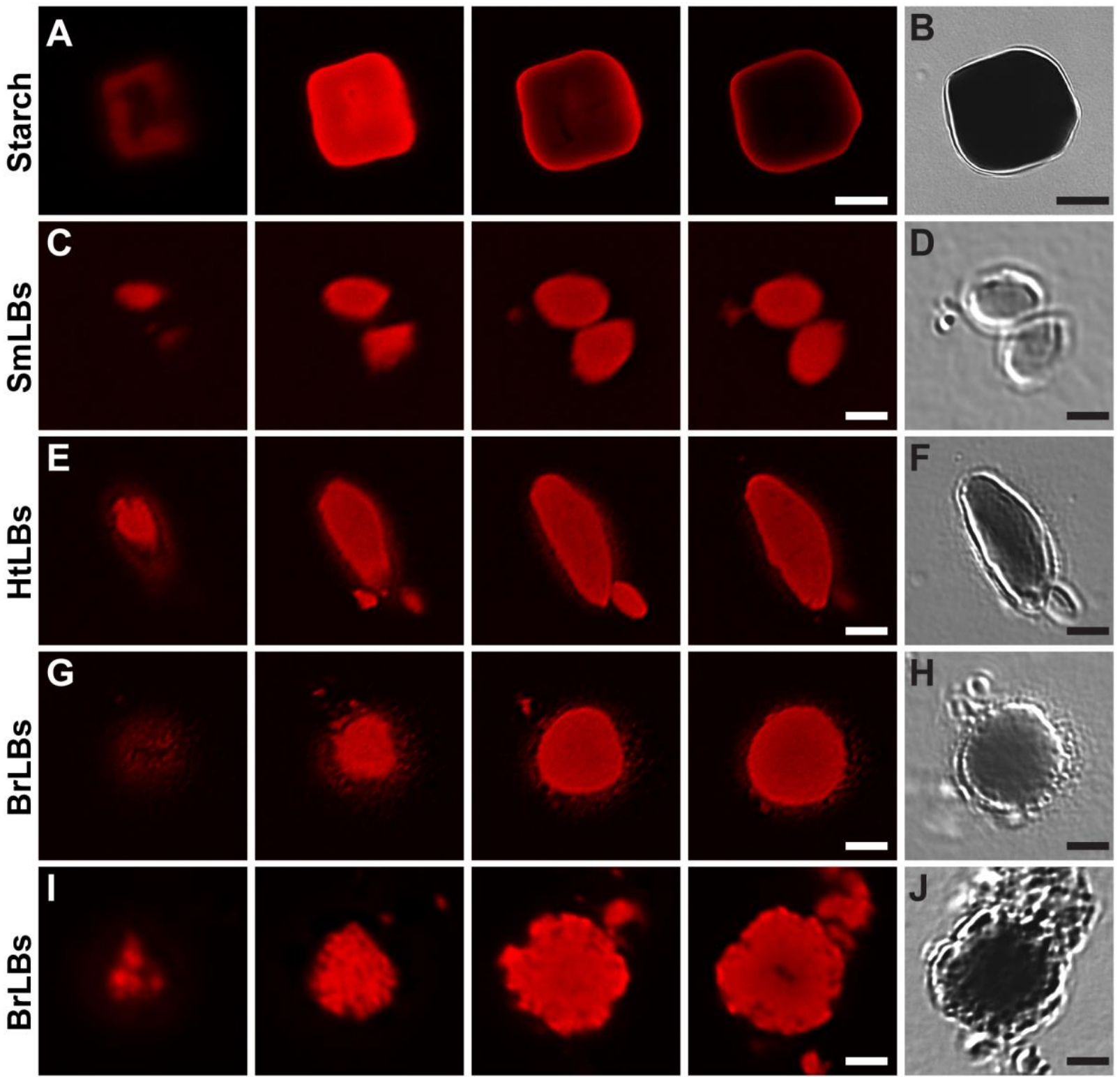

293 Figure 1. Representative $\mathrm{z}$-stack confocal images via the $561 \mathrm{~nm}$ laser line of iodine-stained 294 starch (A), SmLBs (C), HtLBs (E), and BrLBs (G,I). Differential interference contrast (DIC) 295 is also shown for each: starch (B), SmLBs (D), HtLBs (F), and BrLBs (H,J). In (A) and (B), 296 scale bar $=10 \mu \mathrm{m}$ and TRITC panels represent $2 \mu \mathrm{m}$ steps. In (C) through (I), scale bar $=2$ $297 \mu \mathrm{m}$. For SmLBs (C), TRITC panels represent $0.4 \mu \mathrm{m}$ steps. For HtLBs (E) and BrLBs (G, I) 298 TRITC panels represent $0.9 \mu \mathrm{m}$ steps. 

morphology and are typically 1-3 $\mu \mathrm{m}$ in length (Brewer et al., 2019). Like starch, they also

302 fluoresce brightly with $561 \mathrm{~nm}$ excitation after iodine staining, but they display staining

303 throughout the LB, suggesting their smaller size and less dense interior facilitates greater

304 penetration of the triiodide ions (Figure 1C and 1D, Figure S1E through H). LBs isolated

305 from Epm2a-^ hearts (HtLBs) are larger in size (typically 2-4 $\mu \mathrm{m}$, up to $10 \mu \mathrm{m}$ in length) and more elongated than SmLBs (Brewer et al., 2019). They were also stained homogeneously with iodine and were comprised of a visible interior texture and a brighter rim (Figure 1E, Figure S1I and S1K). The textured surface of the LBs was evident using DIC and consistent

309 with what we previously observed via scanning electron microscopy (Figure $1 \mathrm{~F}$ and $1 \mathrm{H}$ )

310 (Brewer et al., 2019). Brain LBs (BrLBs) were also larger in size (typically 2-4 $\mu \mathrm{m}$, occasionally $>10 \mu \mathrm{m}$ in diameter) and varied in morphology more than SmLBs and HtLBs.

312 Some BrLBs were very round and homogenously stained, with a bright rim and textured 313 appearance, similar to HtLBs except that they are not typically elongated (Figure $1 \mathrm{G}$ and

314 1H). Some BrLBs had a very jagged edge with a dense core that did not stain well with

315 Lugol's iodine (Figure 1I and 1J). Very small iodine-positive granules were also evident around LBs from heart and brain (Figure 1 and Figure S1), consistent with previous observations describing “dust-like particles” (Brewer et al., 2019; Van Hoof \& Hageman-Bal, 1967). Overall, the fluorescence produced from the interaction of LBs with the triiodide ions suggests they possess crystalline regions like plant starch. In general, the LBs are more permeable to the triiodide ions than starch, indicating less compact and/or less orderly architecture. However, the lack of central iodine fluorescence in some of the BrLBs (Figure 


\subsection{LBs contain B-type crystallites lacking long-range order}

Unlike glycogen, starch granules possess both short- and long-range order, which can be measured by X-ray scattering techniques. Long-range order refers to the alternation of crystalline and amorphous layers within the growth rings of the starch granule (i.e. lamellae), with a repeat distance of 8-10 nm (Figure 2A, panels $I$ and II) (Blazek \& Gilbert, 2011;

330 Lourdin et al., 2015). Small-angle X-ray scattering (SAXS) is better suited for measuring these nanometer-sized distances (Figure S2A) (Donald, Kato, Perry, \& Waigh, 2001). Shortrange order refers to repeating units on the sub-nanometer scale, i.e. the crystalline organization of double helices formed by the short linear segments of amylopectin (Figure 2A, panels $I I I$ and $I V$ ). Wide-angle X-ray scattering (WAXS) is used to probe sub-nanometersized distances and has been widely utilized to study crystallinity in starch and other polymers (Figure S2A) (Blazek \& Gilbert, 2011; Donald et al., 2001). In starch, amylopectin double helices crystallize in two different allomorphs depending on the starch source (e.g. potato or corn). Typically, A-type is found in cereals while B-type occurs in tubers and highamylose starch (Bertoft, 2017; Qiao et al., 2017). The allomorphic composition can be

340 determined from X-ray diffraction patterns. The double helices of A-type crystals are densely packed into a monoclinic unit cell that contains little water (Figure 2A, panel $V$ ) (Popov et al., 2009). In the B-type crystal, the double helices form a hexagonal network with a central

343 channel that contains water molecules, rendering this allomorph more open and hydrated

344 (Figure 2A, panel VI) (Imberty \& Perez, 1988). We used SAXS and WAXS to characterize 345 the order in native, purified LBs. 

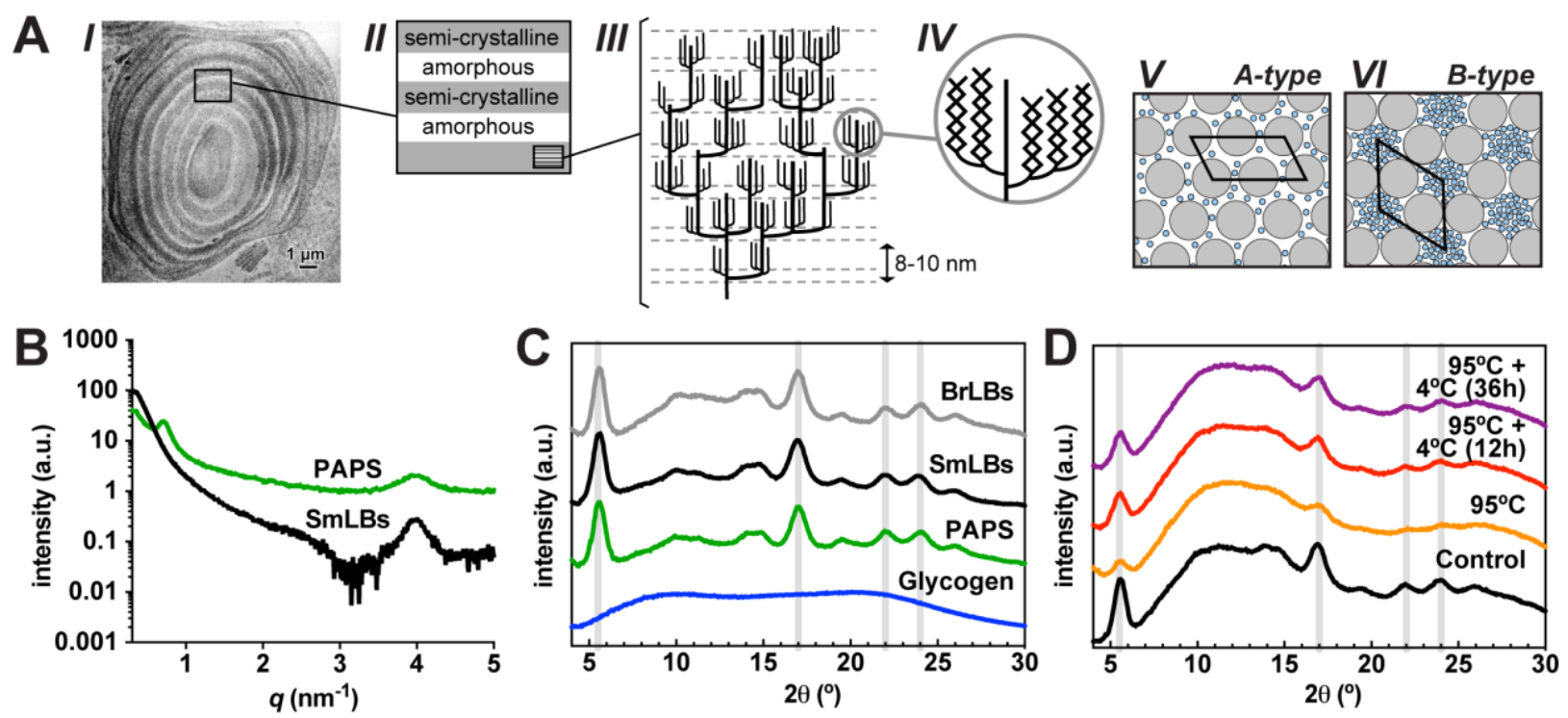

Figure 2. (A) Schematic depiction of long- and short-range order within the starch granule. $I$ : An ultrathin section of a waxy maize starch granule visualized by transmission electron microscopy (TEM) and showing the typical growth rings. II: Growth rings are composed of alternating amorphous and semicrystalline layers (known as lamellae). III: Semicrystalline lamellae are made up of branched amylopectin molecules with amorphous regions containing the branch points and a crystalline region containing the linear segments forming double helices. The total lamellar thickness equals the so-called repeat distance which is typically 8$10 \mathrm{~nm}$ (Nakamura, 2015). The arrangement of the crystalline clusters is still debated. $I V$ : Long, linear glucan chains (black lines) form double helices that make up the crystalline layers of the starch lamellae. The helical packing determines the allomorphic type. $V$ : In Atype, the helices (grey circles) are closely packed, with some water molecules (blue circles) in-between. VI: In B-type, the helices form a hexagonal array surrounding a water-filled channel (Imberty \& Perez, 1988; Nakamura, 2015; Tester, Karkalas, \& Qi, 2004). Reprinted by permission from Springer Nature: Springer, Crystalline Structure in Starch, Lourdin et al. (C) 2015. (B) SAXS profiles of LBs and potato amylopectin starch (PAPS). (C) WAXS profiles of BrLBs and SmLBs compared to those of PAPS and rabbit liver glycogen. (D) WAXS profiles of SmLBs after heating at $95^{\circ} \mathrm{C}$ and cooling down to room temperature, and then with a retrogradation period at $4{ }^{\circ} \mathrm{C}$ either 12 or $36 \mathrm{~h}$ after heating at $95{ }^{\circ} \mathrm{C}$. Grey lines denote the location of characteristic B-type reflections in WAXS profiles. The corresponding 2D SAXS and WAXS patterns are shown in Figure S3.

We first performed SAXS on SmLBs. As a control, we also analyzed a genetically modified starch engineered to contain exclusively amylopectin and no amylose called potato amylopectin starch (PAPS) (Svegmark et al., 2002). PAPS produced a characteristic SAXS profile with two distinct peaks (Figure 2B and Figure S3A). The peak at $q=4.1 \mathrm{~nm}-1$ corresponds to a $d$-spacing of $1.53 \mathrm{~nm}$ and is equivalent to the innermost reflection of the $\mathrm{B}$ - 
375

376

377

378

379

380

381

382

383

384

385

386

387

388

389

390

391

392

393

394

395

396

397

398

type allomorph in a WAXS profile. It corresponds to the spacing of (100) planes in the unit cell (Figure S2A). The reflection at $q=0.76 \mathrm{~nm}-1$ corresponds to a repeat distance of $8.3 \mathrm{~nm}$ (Figure 2B), i.e. the lamellar repeat in the amylopectin structure (Figure 2A, panel III) (Blazek and Gilbert 2011). While the profile of SmLBs also contained the (100) reflection of B-type, the peak corresponding to the lamellar repeat was absent (Figure 2B and Figure S3B). This result indicates that SmLBs do not contain any lamellar units like starch granules. To establish whether LBs are crystalline, we studied them using WAXS with PAPS as a control. We analyzed both SmLBs and BrLBs to determine whether there are allomorphic differences between LB types. PAPS, SmLBs and BrLBs all produced a WAXS diffraction pattern containing characteristic reflections of the B-type allomorph at $2 \theta=5.6,17,22$ and $24^{\circ}$ (Figure 2C, S3C, S3D and S3E) (Buléon, Bizot, Delage, \& Pontoire, 1987). The WAXS reflection at $2 \theta=5.6^{\circ}$ corresponds to the 100 reflection, a typical signature of allomorph $\mathrm{B}$ (Svegmark et al., 2002). Using Equation (1), $d$-spacings were calculated from these values of $2 \theta$ and are in agreement with those of B-type potato starch (Table 1, also see Figure S2B) (Cleven, Van den Berg, \& Van Der Plas, 1978). In contrast, rabbit liver glycogen can be considered amorphous since its WAXS profile does not contain any distinct peak (Figure 2C).

Table 1. $d$-Spacings (in $\mathrm{nm}$ ) calculated from the position (diffraction angle $2 \theta$ ) of the diffraction peaks in the WAXS profiles of PAPS and LB samples using Equation (1) compared to published values (*from Cleven et al. 1978).

\begin{tabular}{lcccc}
\hline Peak \# & 1 & 2 & 3 & 4 \\
\hline PAPS & 1.59 & 0.52 & 0.40 & 0.37 \\
SmLBs & 1.59 & 0.52 & 0.40 & 0.37 \\
BrLBs & 1.59 & 0.52 & 0.40 & 0.37 \\
Potato* & 1.58 & 0.53 & 0.40 & 0.37 \\
\hline
\end{tabular}


When starch is heated in water, it swells and amylose leaches out of the granule. The

401 peak in the SAXS profile disappears, indicating that the lamellae organization is disrupted

402 and no longer present. The peaks in the WAXS profile concomitantly disappear due to the

403 complete loss of crystal structure, i.e. glucan chains are no longer oriented in a repeating

404 helical units (Cameron \& Donald, 1992). However, after gelatinization, as the starch cools

405 down to room temperature, amylose and amylopectin regain B-type crystallinity, a process

406 known as retrogradation. Double helices form from intra- and intermolecular interactions,

407 and aggregate into networks (Putaux, Buleon, \& Chanzy, 2000). However, in retrograded

408 starch, the lamellar periodicity of the native granule does not return (Cameron \& Donald,

409 1991). To test the effect of heat denaturation of LBs, we heated SmLBs at $95^{\circ} \mathrm{C}$, analyzed

410 them by WAXS, and observed that the B-type reflections were significantly reduced (Figure

411 2D). However, when the samples were incubated for 12 or $36 \mathrm{~h}$ at $4{ }^{\circ} \mathrm{C}$ post-heating, the

412 reflections gradually reappeared (Figure 2D). These data indicate that solubilized LBs

413 structurally behave like retrograding starch: crystallinity is reduced upon heating but

414 reappears spontaneously in a B-type arrangement after incubation at low temperature.

\subsection{Microscopic effects of mechanical, thermal and chemical treatments on LBs}

417 Thus far, the data demonstrate that LBs are comprised of B-type crystallized glucan

418 chains. As discussed in the introduction, the Pflüger method involves both thermal and

419 chemical treatment resulting in the conversion of micron-sized LBs to nanometer-sized

420 particles. Since modification of starch and other polysaccharides by mechanical, thermal,

421 chemical, or enzymatic means provides structural insights, we sought to explore the effects of

422 multiple treatments on LB structure by light microscopy and iodine staining.

423 Untreated SmLBs are uniformly small and ovoid and stain intensely with Lugol's iodine

424 (Figure 3A). We found that mild sonication (5 x $5 \mathrm{~s}$ at $20 \mathrm{kHz}$ with $25 \%$ amplitude) disrupted 
425 SmLB structure almost entirely (Figure 3B). After this treatment, most of the LBs were 426 fragmented into clumped, lace-like networks of polyglucosan material that lightly stained 427 with Lugol's. Only a few intact SmLBs were left associated with the lace-like networks 428 (Figure 3B, inset). This result is quite different from what has been observed with starch 429 granules: treatment of starch granules with ultrasonication for minutes or longer produces 430 only mild disruption of granular structure (Majzoobi, Seifzadeh, Farahnaky, \& Mesbahi, $4312015)$.

432 We next examined the effect of heat denaturation on LB structure. SmLBs were heated 433 in water for $30 \mathrm{~min}$ at $95^{\circ} \mathrm{C}$ and were completely converted to lacy networks (Figure 3C).

434 The resulting material after heating appeared very similar to the material produced by 435 sonication, except that no intact SmLBs were observed after the heat treatment. The clumps 436 of material were even more dispersed with longer heating $\left(2 \mathrm{~h}\right.$ at $\left.95^{\circ} \mathrm{C}\right)$, and very small, 437 freely floating particles with diameters less than $1 \mu \mathrm{m}$ were more abundant (Figure 3D). 438 Since the Pflüger method is the standard technique used to precipitate glycogen and has 439 been often employed to purify polysaccharides from LD mice (Sullivan et al., 2019; 440 Tagliabracci et al., 2008), we next analyzed the effects of this treatment on SmLB structure. 441 In this method, the tissue is boiled in $30 \% \mathrm{KOH}$ for $2 \mathrm{~h}$, the polysaccharide is precipitated 442 three times with 2 volumes of cold ethanol, and then it is re-dissolved in water. $\mathrm{LiCl}$ is added 443 with the ethanol to ensure complete precipitation (Tagliabracci et al., 2008). The successive 444 precipitation steps remove impurities and residual $\mathrm{KOH}$, and the resulting individual particles 445 can be analyzed by TEM or SEM (Sullivan et al., 2019; Tagliabracci et al., 2008). To test the 446 contribution of the different portions of the Pflüger method on LB structure, we performed 2

$447 \mathrm{~h}$ heat treatments in either water (i.e. hydrothermal) or $30 \% \mathrm{KOH}$, followed by ethanol 448 precipitation. The SmLBs that were precipitated with ethanol and $\mathrm{LiCl}$ three times following 

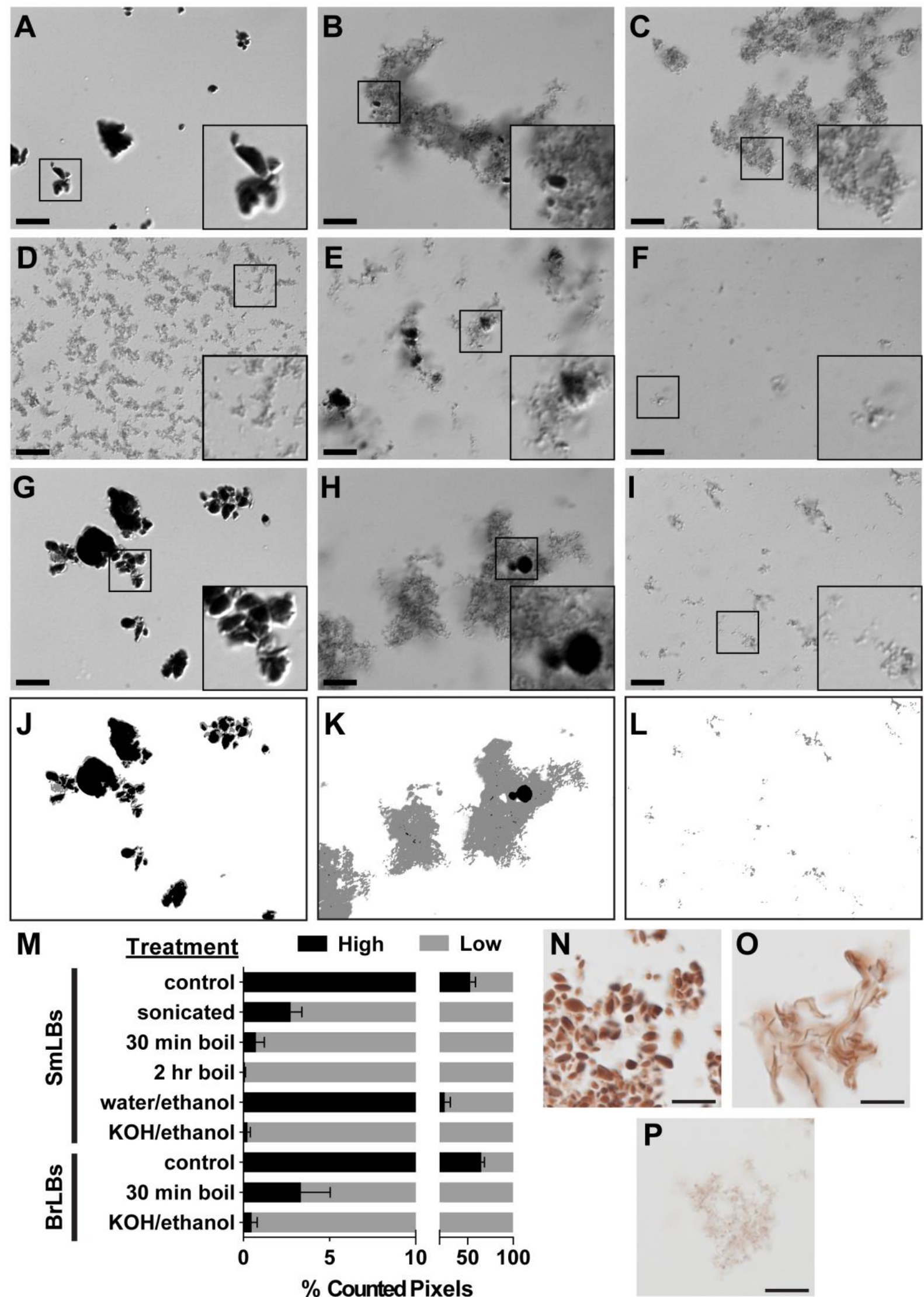

$\%$ Counted Pixels

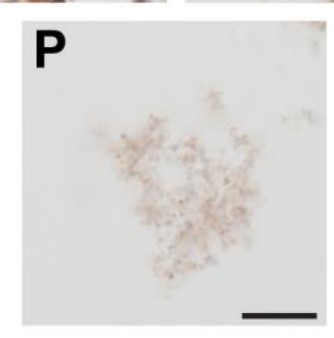

450 Figure 3. Effects of multiple treatments on the structure of LBs. LBs were stained with

451 Lugol's iodine after each treatment and visualized by light microscopy. Control SmLBs (A); 452 mildly sonicated SmLBs (B); SmLBs heated in water at $95{ }^{\circ} \mathrm{C}$ for $30 \mathrm{~min}(\mathrm{C})$; SmLBs heated 
in water at $95{ }^{\circ} \mathrm{C}$ for $2 \mathrm{~h}$ (D); SmLBs heated in water at $95{ }^{\circ} \mathrm{C}$ for $2 \mathrm{~h}$, then precipitated 3 times with 2 volumes cold ethanol and $\mathrm{LiCl}(\mathrm{E})$; SmLBs treated with the Pflüger method ( $2 \mathrm{~h}$ at $95{ }^{\circ} \mathrm{C}$ in $\left.30 \% \mathrm{KOH}\right)(\mathrm{F})$. Control BrLBs $(\mathrm{G})$; BrLBs heated in water for $30 \mathrm{~min}(\mathrm{H})$; BrLBs treated with the Pflüger method (I). Thresholding was used to quantitate LBs (with high intensity staining) and polyglucosan (with low intensity staining) in micrographs. Examples of applied thresholds for $(G)$ through (I) are shown in $(J)$ through (L). Pixels with high intensity (grey values 0-50) and low intensity (grey values 51-140) were counted and shown as a percentage of total counted per micrograph. Pixels with grey values above 141 were considered background and not counted. The values shown are the averages of 9 micrographs \pm standard error. (N, O, P) Structural transition of SmLBs during heating. Control SmLBs (N), SmLBs heated for 1 minute and flash frozen (O), and SmLBs heated for 5 min and flash frozen $(\mathrm{P})$. After freezing, samples were thawed, stained with Lugol's iodine, and visualized using a light microscope with a Zeiss 512 color camera. All scale bars $=10$ $\mu \mathrm{m}$. Each inset is a $2.5 \mathrm{x}$ magnification of the boxed region.

a 2-h boil in water were more difficult to resuspend in water: the white pellet did not easily go into solution, and flakes of polysaccharide were visible. Using light microscopy, this material contained intensely stained aggregates, filamentous structures and granular particles (Figure 3E). The aggregates were more amorphous and less compact than control SmLBs, but stained with a similar intensity, suggesting that the polyglucosan became re-crystallized with precipitation. However, the LBs that underwent precipitation after a 2-h hydrothermal treatment did not reform into the compact, ellipsoidal shape of native SmLBs (Figure 3F). In contrast, $\mathrm{LBs}$ that were boiled in $30 \% \mathrm{KOH}$ for $2 \mathrm{~h}$ and then precipitated three times with ethanol and $\mathrm{LiCl}$ were very easily re-suspended in water and produced no visible precipitate. This material did not contain intensely-staining aggregates with Lugol's solution and only a few small clumps of polyglucosan material were visible (Figure 3F). Thus, boiling in $\mathrm{KOH}$ instead of water fully dissociates the LBs into polyglucosan particles rather than networks, and in these alkaline conditions, the polyglucosan is much less prone to re-aggregate upon ethanol/LiCl precipitation.

Hydrothermal and Pflüger treatments were also applied to BrLBs and the products were visualized via light microscopy. As expected, in the absence of treatment BrLBs were larger 
and more irregularly shaped than SmLBs and stained with Lugol's iodine (Figure 3G). A 30min hydrothermal treatment partially converted BrLBs to polyglucosan networks, though some BrLBs remained intact, surrounded by the lacy material (Figure $3 \mathrm{H}$ ). As with the SmLBs, Pflüger treatment released a large amount of small granular particles, but more lacy polyglucosan was still present in these samples compared to the Pflüger-treated SmLBs (Figure 3I versus 3F). These samples were also easy to re-suspend after precipitation. Image thresholding was used to quantitate LBs, LB-like aggregates and the lace-like polyglucosan in treated samples. In the recorded micrographs, intact LBs were very dark grey or black, with grey values of 50 or less (Figure 3J and K). Polyglucosan networks or particles appeared in lighter shades of grey, with values between 51 and 140 (Figure 3K and L). Pixel counts from multiple micrographs showed that $>95 \%$ high intensity staining (i.e. intact LBs) were disrupted by sonication and heating in water for $30 \mathrm{~min}$ (Figure 3M). No intact LBs were found in the SmLB samples that were heated in water for $2 \mathrm{~h}$ or the Pflüger-treated samples. Interestingly, the high intensity observed in control samples (53\% of counted pixels) could be partially restored by ethanol treatment after heating for 2 -h in water $(25 \%$ of

500 counted pixels). These results demonstrate that crystalline LBs undergo a structural transition to polyglucosan networks in the presence of heat, and they can be fully dissociated with the Pflüger method. Without $\mathrm{KOH}$, ethanol precipitation causes polyglucosan to re-aggregate and form dense, LB-like structures.

To visualize the transition from native LB to lacy polyglucosan networks, SmLBs were 505 heated at $95^{\circ} \mathrm{C}$ for 1 or $5 \mathrm{~min}$ in water, immediately flash frozen in liquid nitrogen, thawed, and stained with Lugol's iodine. Control, native LBs stained bright reddish-brown in Lugol's iodine (Figure 3N). Samples that were heated for 1 min displayed numerous amorphous, redbrown gelatinous structures in addition to small fragments of lightly stained lace-like 
gelatinous structures were observed (Figure 3P). These results are strongly reminiscent of

511 gelatinization, the irreversible order-disorder transition that starch undergoes when heated in

512 water characterized by swelling, increased viscosity and a loss of crystallinity (Ratnayake \&

513 Jackson, 2006). These results strongly suggest that like starch, LBs gelatinize at high

514 temperatures.

515

\subsection{TEM observation of hydrothermally-treated and lintnerized LBS}

Normal glycogen particles can easily be visualized using transmission electron microscopy (TEM) (Revel, Napolitano, \& Fawcett, 1960; Ryu et al., 2009). To study the structure of disrupted LBs with higher resolution than can be achieved by the light microscope, the hydrothermally-treated SmLBs were analyzed by TEM. After heating samples for $30 \mathrm{~min}$ at $95{ }^{\circ} \mathrm{C}$, they were allowed to cool to room temperature, stained with uranyl acetate, and visualized. Two types of species were observed: i) individual spheroidal particles with diameters ranging from 15 to $25 \mathrm{~nm}$ and appearing similar to typical glycogen granules (Ryu et al., 2009) and ii) knobby worm-like objects (Figure 4A). The worm-like objects had widths varying between 5 and $15 \mathrm{~nm}$, bearing a strong resemblance to retrograded amylopectin (Putaux et al., 2000). BrLBs were also visualized by TEM after a 30-min hydrothermal treatment (Figure 4B). The individual sponge-like particles were slightly smaller in size (12 to $23 \mathrm{~nm}$ ), irregular, and difficult to distinguish; the worm-like objects were also present, with a similar width to those of the SmLBs. After heating, the

530 SmLB and BrLB samples were also incubated at $4{ }^{\circ} \mathrm{C}$ to determine whether the particles would aggregate into networks, i.e. retrograde, in a manner similar to amylopectin. Indeed, after $72 \mathrm{~h}$, larger networks formed that appeared to be aggregates of the worm-like elements observed after the initial solubilization at $95{ }^{\circ} \mathrm{C}$ (Figure 4C and 4D). However, individual

534 glycogen-like particles that did not aggregate in the SmLBs samples were still observed 

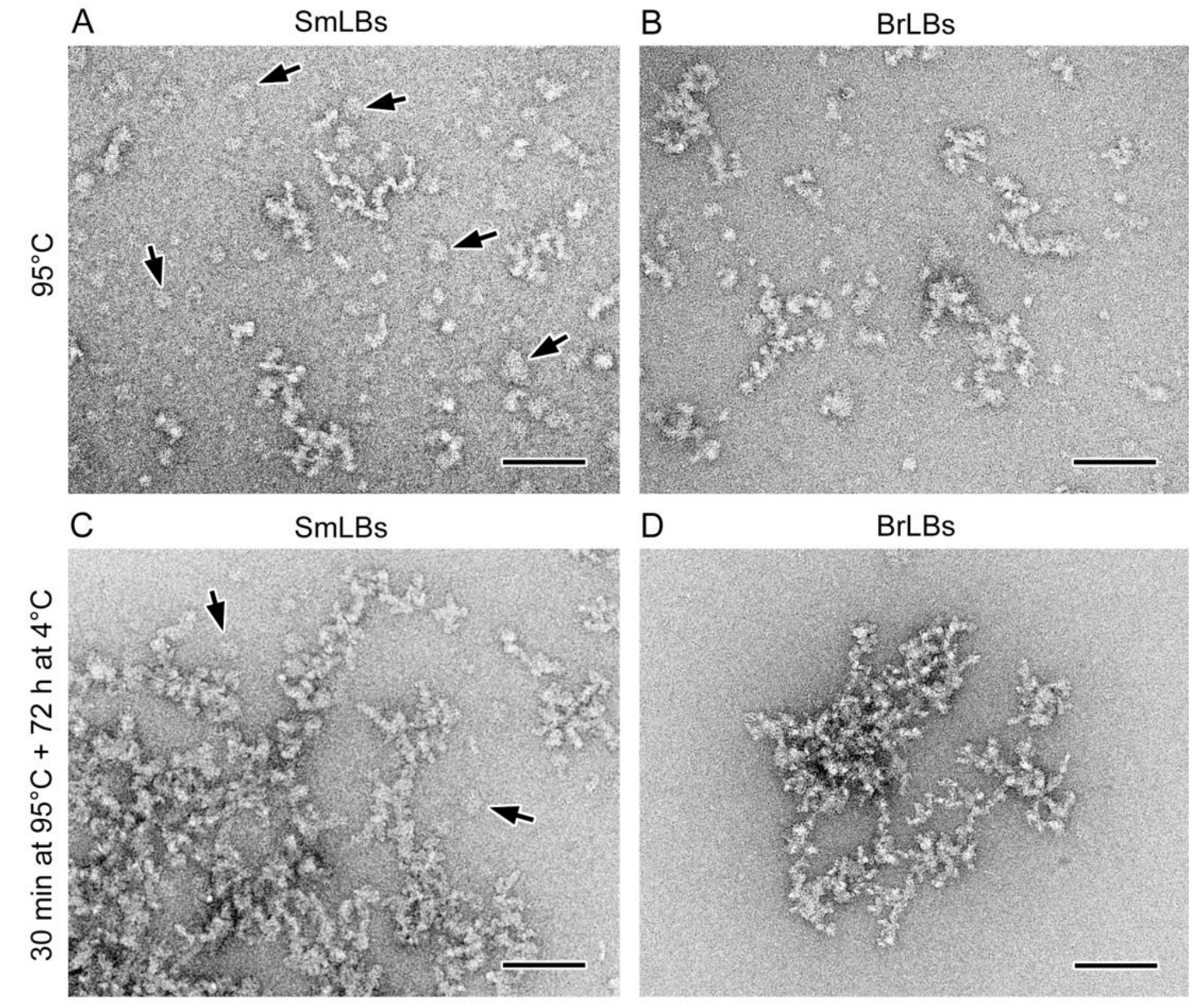

E

$\mathrm{F}$
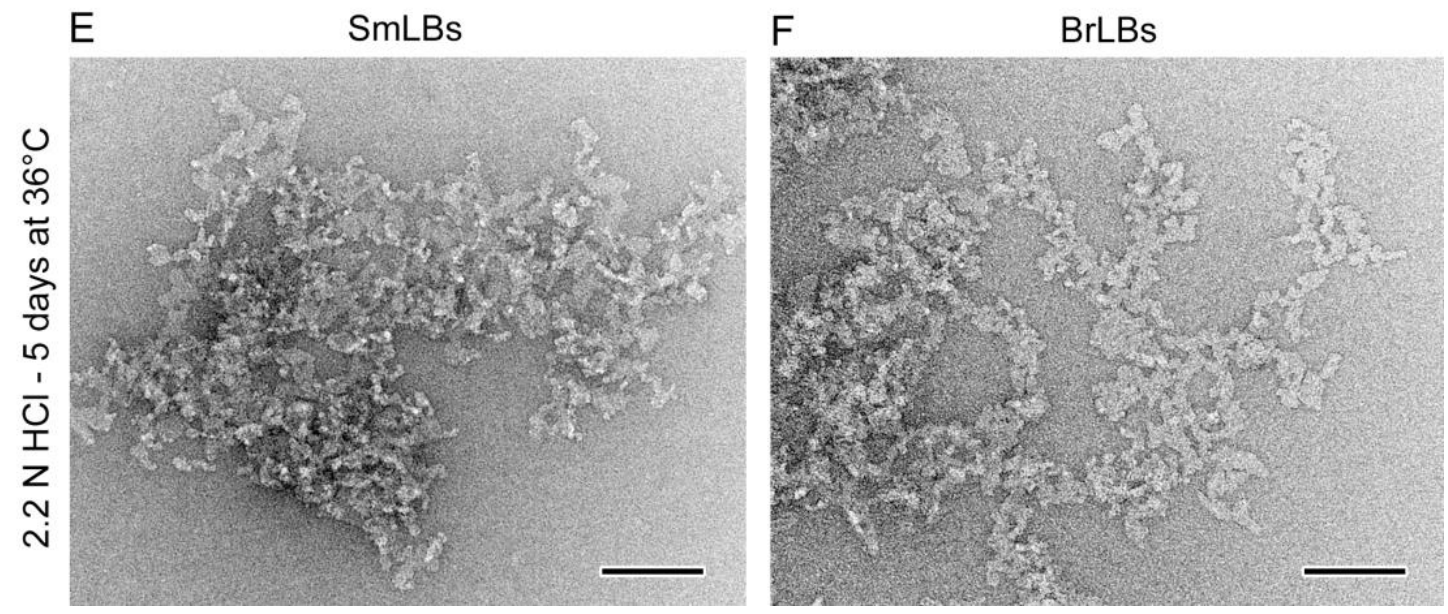

536 Figure 4. Transmission electron microscopy (TEM) observation of LB structure. (A, B, C, D) 537 TEM images of negatively stained specimens prepared by heating dilute aqueous SmLB (A, $538 \mathrm{C}$ ) and $\operatorname{BrLBs}(\mathrm{B}, \mathrm{D})$ suspensions at $95{ }^{\circ} \mathrm{C}$ for $30 \mathrm{~min}$. The specimens were observed 539 immediately after cooling them to room temperature (A, B) and after 3 days $(72 \mathrm{~h})$ at $4{ }^{\circ} \mathrm{C}(\mathrm{C}$, 540 D). (E, F) Images of negatively stained specimens prepared by hydrolyzing LBs in $2.2 \mathrm{~N} \mathrm{HCl}$ 541 for 5 days at $36^{\circ} \mathrm{C}$ : (E) SmLBs; (F) BrLBs. Scale bars $=100 \mathrm{~nm}$. 
543 (Figure S4). Interestingly, retrograded amylopectin takes on a very similar necklace-like

544 appearance where over time the molecules associate to form networks (Putaux et al., 2000).

545 These data are consistent with the re-formation of B-type crystallinity after a cooling period

546 post-heating (Figure 2D). Like amylopectin, polyglucosan particles of LBs can retrograde,

547 i.e. spontaneously form crystalline associations via intermolecular helical interactions to form

548 a network or necklace-like pattern.

549 Lintnerization is the process of treating starch granules with mild $\mathrm{HCl}$ at sub-

550 gelatinization temperatures for long periods of time (Bertoft, 2017; Jacobs et al., 1998). The

551 amorphous regions of the starch granule hydrolyze early during lintnerization, while

552 crystalline regions are left intact. This effect is due to the protection of glucose residues and

553 glycosidic linkages within the crystalline lamellae containing the double helices, which are

554 less easily penetrated by the hydrogen ions (Jacobs et al., 1998; Putaux, Molina-Boisseau,

555 Momaur, \& Dufresne, 2003). The morphology and properties of the lintners, the insoluble

556 residues after hydrolysis, vary with species, amylose content, and starch allomorph

557 (Goldstein et al., 2016; Putaux et al., 2003; Wikman et al., 2014). This process allows the

558 crystalline residues of the starch to be analyzed in isolation.

559 BrLBs and SmLBs were treated with $2.2 \mathrm{~N} \mathrm{HCl}$ for 5 days at $36^{\circ} \mathrm{C}$, washed with water

560 and imaged by TEM after negative staining. The BrLB and SmLB lintners were very similar

561 in morphology, both appearing as lacy networks of flat elements (Figure 4E and 4F). Similar

562 lacy networks have been reported for lintnerized potato starch, particularly waxy potato

563 starch, which exhibit B-type crystallinity (Wikman et al., 2014). This result is in contrast to

564 A-type waxy maize starch or barley starch lintners that form well-defined polyhedral platelet

565 nanocrystals, reflective of A-type helix packing (Goldstein et al., 2016; Putaux et al., 2003). 
When visualized by TEM, normal glycogen particles typically appear as individual

569

570

571

572

573

574

575

576

577

578

579

580

581

582

583

584

585

586

587

588

589

590

591

592 granules ranging from 20 to $50 \mathrm{~nm}$ in diameter, but can form large $\alpha$ rosettes in the liver (Ryu et al., 2009). Heated SmLBs contained individual particles from 15 to $25 \mathrm{~nm}$ in diameter, which appeared similar to normal glycogen (Figure 4A and 4C). However, heated SmLBs and BrLBs both contained worm-like elements with a diameter of 5-15 $\mathrm{nm}$ after heating and unambiguous B-type lintners after treatment with mild acid. Such structures are quite distinct from the normal structure of glycogen and may result from an aggregation of very small or very long polysaccharide particles.

To determine the distribution of particle sizes within the LB preparations, native SmLBs, BrLBs and HtLBs were dissociated into polyglucosan particles via the Pflüger method and then analyzed using size exclusion chromatography (SEC). Rat muscle glycogen was used as a reference for normal glycogen particle distribution, with a single peak corresponding to particles with hydrodynamic radius $(R \mathrm{~h}) \sim 10-20 \mathrm{~nm}$ (Figure 5). All LB samples displayed a very narrow peak at $R \mathrm{~h} \sim 1 \mathrm{~nm}$, likely too small to be a polyglucan (estimated to be the approximately the same size as an oligosaccharide or peptide). Beyond this peak, SEC data for SmLB particles were consistent with size distributions of polysaccharide from Epm $2 a-/$ skeletal muscle as previously published (Sullivan et al., 2019): a primarily bimodal distribution with the first major peak at hydrodynamic radius $(R \mathrm{~h}) \sim 3 \mathrm{~nm}$ and the second at $R \mathrm{~h}$ $\sim 10-15 \mathrm{~nm}$. The second peak is consistent with the size of rat muscle glycogen particles and previous reports of glycogen from various sources (Sullivan et al., 2019; Sullivan et al., 2014). The polyglucosan particles from BrLBs and HtLBs, however, lacked the peak at $R \mathrm{~h}$ $\sim 10-15 \mathrm{~nm}$, and contained only a major peak at $R \mathrm{~h} \sim 3 \mathrm{~nm}$ (Figure 5). These striking results indicate that pure SmLBs contain a variety of particle sizes, with an enrichment of particles comparable in size to glycogen and very small particles. In contrast, dissociated BrLBs and HtLBs contain exclusively very small particles, which are likely to form early during 
glycogen synthesis (Sullivan et al., 2019). The worm-like aggregates and networks that were

594

595

596

597

598

599

600

601

602

603

604

605

606

607

608

609

610

611

612

613

614

615

observed after heating via TEM (Figure 4A, B, C and D) are likely to be aggregates of the small particles with $R \mathrm{~h} \sim 3 \mathrm{~nm}$ (i.e. roughly $6 \mathrm{~nm}$ in diameter), since retrogradation and reaggregation can rapidly start upon cooling the suspension (Michen et al., 2015). The absence of the individual glycogen-like particles in the BrLB samples in Figure 4 is consistent with the SEC data.

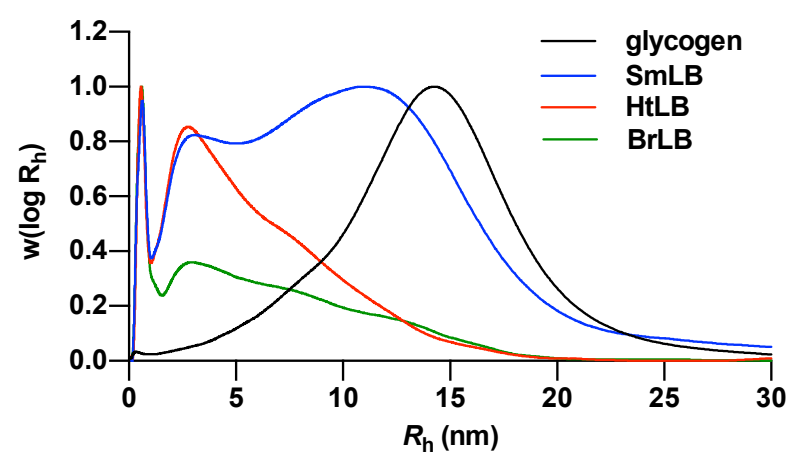

Figure 5. SEC weight distributions, $w(\log R \mathrm{~h})$ of $\mathrm{KOH}$-dissociated polyglucosan particles from BrLBs (green), HtLBs (red) and SmLBs (blue), as a function of hydrodynamic radius, $R \mathrm{~h}$. Rat muscle glycogen was used as a reference (black). Normalization is arbitrary with SEC weight distributions and was chosen to be at the peak with an $R \mathrm{~h}$ of $\sim 1 \mathrm{~nm}$.

\section{Discussion}

\subsection{Proposed model for LB architecture and formation}

We describe three levels of polyglucosan architecture in our study: the native LB, the polyglucosan network, and the polyglucosan particle. The largest entity is the native LB, typically ranging in diameter from 2-10 $\mu \mathrm{m}$ (Figure 6). In heart and brain, LBs consist only of very small polyglucosan particles, while in skeletal muscle, LBs contain a mixture of glycogen-like particles and very small polyglucosan particles. Minimal branching favors Btype crystal packing, where double helices form a hexagonal unit with a water-filled interior channel. It is possible that some double helices are formed from adjacent chains within the same polyglucosan molecule (intramolecular interactions), while others may be formed from 


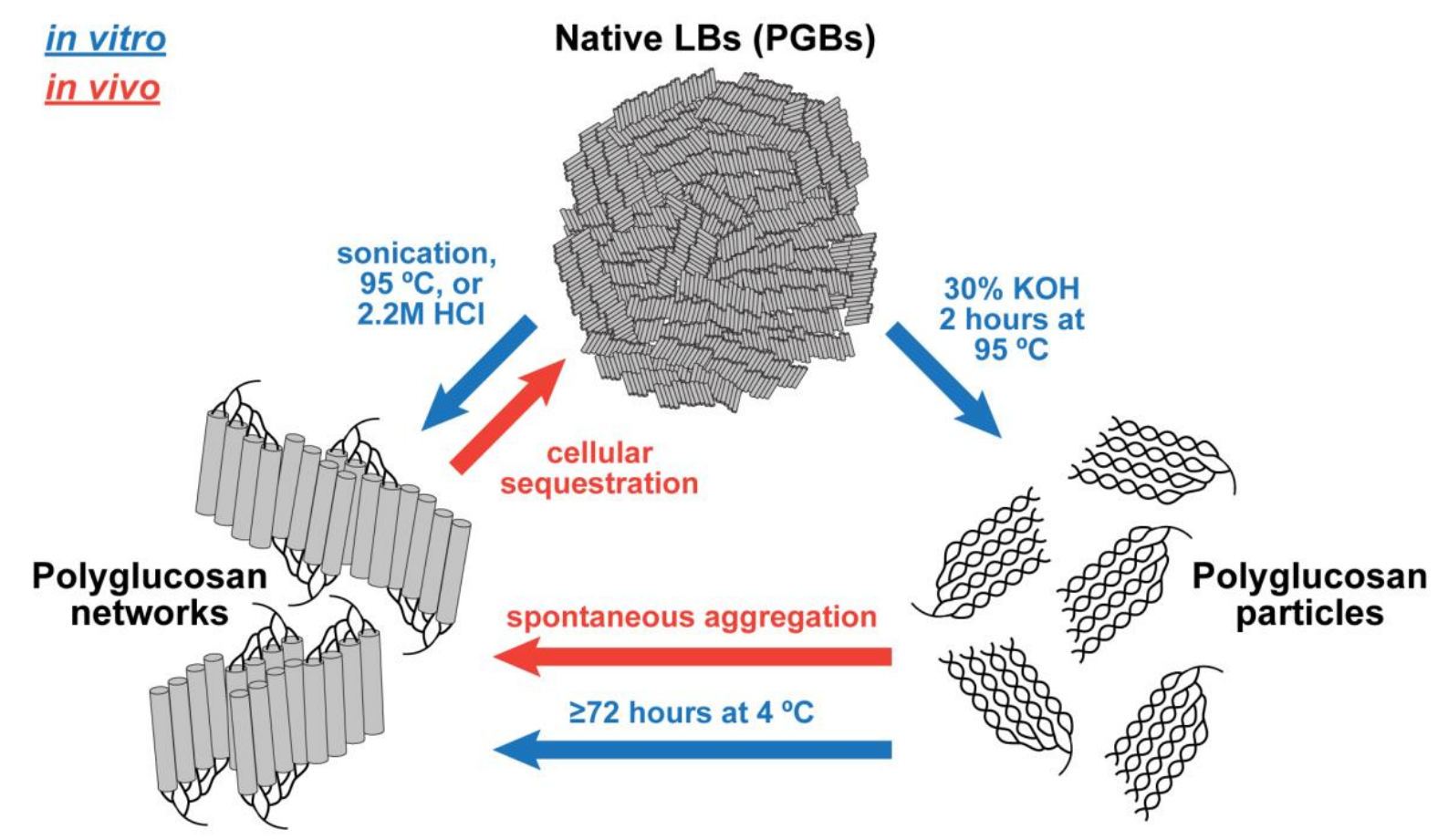

Figure 6. A model for LB structure and formation, as in heart and brain. Blue arrows and text

619 illustrate what was observed in vitro, and red arrows and text illustrate an in vivo model. LBs

620 are primarily comprised of very small polyglucosan particles with abnormally long glucan

621 chains that can crystallize into the B-type allomorph. LBs (i.e. intact PGBs) can be disrupted

622 into networks by sonication, heat, or acid, and the individual particles can be completely

623 dissociated via the Pflüger method. Aggregation of these polyglucosan particles into

624 networks is likely to occur spontaneously in vivo since we observed spontaneous

625 retrogradation in vitro after 72 hours at $4{ }^{\circ} \mathrm{C}$. Since LBs have a very defined structure, active

626 cellular sequestration is likely responsible for the distinct tissue-specific morphologies. LBs

627 in skeletal muscle also contain glycogen-like particles (not shown).

628

629

630 chains from two different polyglucosans (intermolecular interactions). For most LB types,

631 crystalline units are randomly arranged, in stark contrast to the lamellar structure of starch

632 granules. The only exception is BrLBs where in some granules iodine did not penetrate to the

633 center. These results are consistent with previous TEM studies suggesting some LBs are

634 composed of radially arranged fibrils and a dense core (Berard-Badier et al., 1980; Cavanagh,

635 1999; Ishihara et al., 1987). 
The second structural entity is the polyglucosan network that emerges when intact LBs are heated, mechanically agitated, or lintnerized (Figure 6). These networks resemble potato lintners and retrograded (i.e. re-precipitated) amylopectin, both of which exhibit B-type crystallinity (Putaux et al., 2000; Wikman et al., 2014). Since amylopectin and LD polysaccharide are both infrequently branched polysaccharides with long glucan chains capable of crystallizing, it is not surprising that the two polysaccharides would associate in a similar network formation. The networks are composed of knobby fibers between 5 and 20 $\mathrm{nm}$ in diameter. The morphology has been described as resembling a necklace, where the "pearls" are laterally packed helical bundles connected by longer amylopectin chains (Putaux et al., 2000). In the case of LD, it is likely that molecules associate via intermolecular helical interactions to form these necklace-like fibers (Figure 6). presumably an aberrantly branched glycogen $\beta$-particle with such long chains that it crystallizes. We have shown that polyglucosan particles are best dissociated with Pflüger treatment, due to heating in $\mathrm{KOH}$. Indeed, alkaline treatment increases the solubility of both glycogen and starch (Kerly, 1930; Wang et al., 2014), and certain forms of these polysaccharides (desmoglycogen or 'fixed' glycogen and resistant starch) can only be solubilized with alkali (Perera, Meda, \& Tyler, 2010; Stetten, Katzen, \& Stetten, 1958). This phenomenon is likely simply because alkali solutions disrupt hydrogen bonding between hydroxyl groups of neighboring glucosyl units (Thys et al., 2008). Once dissociated, SEC distributions show an enrichment of molecules smaller than typical glycogen particles, most prominently in the brain and heart (Figure 5). Dissociated polyglucosan particles from skeletal muscle are comprised of small molecules of $R \mathrm{~h} \sim 3 \mathrm{~nm}$ (i.e. $\sim 6 \mathrm{~nm}$ in diameter) and also contain molecules similar in size to regular muscle glycogen with a peak $R \mathrm{~h}$ of $\sim 10-15$ $\mathrm{nm}$ (i.e. $20-30 \mathrm{~nm}$ in diameter). These data are in agreement with previous reports while 
661 providing additional insights (Sullivan et al., 2019). These results indicate that glycogen from

662 LD mice may be particularly vulnerable to precipitate early during synthesis of the glycogen

663 granule, before it reaches its normal size $(\sim 25 \mathrm{~nm})$. Dissociated polyglucosan bodies from the

664 skeletal muscle, heart and brain all also contain a peak at an $R \mathrm{~h}$ of $\sim 1 \mathrm{~nm}$ (estimated to be the

665 size of a short oligosaccharide or peptide). Whether this is small carbohydrate or protein that

666 is associated with LBs is unknown and is being further explored.

667 In starch, the crystalline, double helical conformation of glucan chains retards their

668 access by degrading enzymes (Emanuelle et al., 2016). Presumably, the long chains of

669 polyglucosan particles take on a similar conformation and prevent them from being degraded

670 by the glycogenolytic machinery (Sullivan et al., 2017). As a result, polyglucosan particles

671 accumulate, potentially associating into networks inside of cells like the ones we observed in

672 vitro (Figure 6). Since we observed that LBs undergo retrogradation (Figure 2D, 5C and 5D),

673 crystallization and network formation likely occur spontaneously, similar to the natural

674 retrogradation of amylopectin after it is cooked. It is likely that the cell, detecting an

675 unknown and potentially toxic species, sequesters the spontaneously forming polyglucosan

676 networks into non-membrane-bound inclusions that become LBs. LBs have been shown to be

677 decorated with a variety of proteins involved in glycogen metabolism, ER stress, ubiquitin,

678 and autophagy and delineated by cytoskeletal elements in vivo (Criado et al., 2012; Machado-

679 Salas et al., 2012). Thus, the formation of this structural entity may not be entirely

680 spontaneous; cellular mechanisms may assist in polyglucosan sequestration. We showed that

681 ethanol precipitation of LBs after boiling in water led to some polyglucosan clumps that

682 stained as intensely as native LBs. This treatment may in some way resemble the process of

683 active polyglucosan sequestration that occurs in cells. The shape and size of the LB is likely

684 dictated by the internal constraints of the cell type where it forms, but it may also be 
influenced by the CLD of the constituent polyglucosan and/or effects of surrounding

686

687

688

689

690

691

692

693

694

695

696

697

698

699

700

701

702

703

704

705

706

707

708 molecules.

The lack of higher order in LBs is not surprising, given that they are an aggregate that forms in response to an aberrant cellular mechanism. B-type crystallization is favored by longer chains and lower temperatures (Cai \& Shi, 2013; Gidley \& Bulpin, 1987). Even native A-type amylopectin can take on a B-type conformation after retrogradation (Qiao et al., 2017). A previous study showed that the in vitro enzymatic chain elongation of glycogen produced dendritic particles with B-type crystallinity (Putaux, Potocki-Véronese, RemaudSimeon, \& Buleon, 2006). TEM micrographs suggest that over time, double helical segments formed as a result of intra- and intermolecular entanglement of the extended glucan chains. It has been proposed that in LD, the lack of laforin or malin leads to an imbalance in glycogen synthase and branching enzyme activities, causing aberrant chain elongation (Sullivan et al., 2017). Thus, the entanglement and crystallization of artificially elongated glycogen as described by Putaux et al. (2006) may resemble what occurs in vivo in LD.

\subsection{Nomenclature: polysaccharides, glycogen, polyglucosan, PGBs and LBs}

Terminology choices have been carefully considered in this study. The term 'polysaccharide' denotes any polymer of sugar molecules, and thus glycogen, amylopectin, amylose, and polyglucosan are all polysaccharides. The term 'polyglucan' refers only to polysaccharides containing glucose units attached by glycosidic bonds, and more specifically, $\alpha$-polyglucans are those with $\alpha-1,4$ and $\alpha-1,6$ glycosidic bonds. Thus, all of the polysaccharides discussed in this paper are also $\alpha$-polyglucans. However, we abstained from using the term 'polyglucan' due to its similarity to 'polyglucosan' and also since no other types of polysaccharides are discussed. 
710 'glycogen', and LD mice are said to have increased glycogen in their tissues. However,

711 studies of the polysaccharide content of 'insoluble' vs. 'soluble' glycogen fractions in LD

712 mice show that LD mice display an increase in the insoluble fraction, but the soluble fraction

713 (i.e. normal glycogen) is equivalent to wild-type (WT) mice (DePaoli-Roach et al., 2010;

714 Sullivan et al., 2019). The Pflüger purified polysaccharides are actually a mixture of both normal glycogen and abnormal polysaccharides that we refer to herein as polyglucosan. LD tissues have approximately the same amount of soluble glycogen as WT mice, and the total increase in polysaccharide, is exclusively due to insoluble polyglucosan (Sullivan et al.,

718 2019; Sullivan et al., 2017). The polyglucosan of LD is a precipitation-prone polysaccharide with long chains and a molecular size much smaller than the typical glycogen particle that can form B-type crystalline units. This material is quite divergent from the typical definition of glycogen, i.e. a soluble glucose polymer with $\sim 13$ glucose units per chain, regular branching, and an average diameter of about $25 \mathrm{~nm}$ (i.e. 10,000 glucose units) (Brewer \& Gentry, 2019; Roach, 2002). Therefore, the abnormal polysaccharide should be called polyglucosan rather than glycogen.

One should also recognize the difference between 'polyglucosan', 'polyglucosan bodies' (PGBs), and 'LBs'. Polyglucosan refers to any abnormal glucose polymer containing $\alpha$ glycosidic linkages found in mammalian tissues, but its exact chemical characteristics vary with its pathological origin. 'PGBs' refer to the compacted inclusions of polyglucosan observed in vivo, which can be isolated as intact entities (Brewer et al., 2019; Sakai et al., 1969; Yokoi et al., 1968). Corpora amylacea and LBs are considered PGBs, but not all PGBs are exactly equivalent. 'LBs' are the PGBs found in LD, which we have shown are morphologically distinct based on tissue type. Additionally, in this study, we specifically used the term 'disrupt' to refer to any modification of the PGB structure, such as by 
sonication or heating, while 'dissociate' referred to the complete separation of individual polyglucosan particles via the Pflüger method.

736

\subsection{Similarities of LBs with other PGBs and amyloid and their relevance to neurological}

738 disease

739 PGBs of different pathologies are overall very similar but distinguishable by subtle ultrastructural and histochemical qualities. LBs are the only PGB to display a darkly staining central core with radiating fibrils visible by electron microscopy (Cavanagh, 1999). The polyglucosan fibrils in LBs are somewhat thicker and more electron dense than those in the PGBs of GSD IV, a disease caused by a deficiency in glycogen branching enzyme (GBE)

744 (Ishihara et al., 1987). With Lugol's iodine, LBs are brown, corpora amylacea are purplishbrown, and the GSD IV PGBs are distinctly purple, reflecting their abnormally long glucan chains (Reed Jr et al., 1968; Sakai et al., 1969; Sullivan et al., 2019). The PGBs of GSD IV do not stain with toluidine blue, in contrast to corpora amylacea and LBs, suggesting they have a much lower phosphate content (Reed Jr et al., 1968). Furthermore, a recent study reported that polyglucosan from GBE mutant mice (a model of Adult Polyglucosan Body Disease) had normal levels of phosphorylation at the 6-hydroxyls and even longer chains than LD polyglucosan (Sullivan et al., 2019). According to another study, corpora amylacea had even more phosphate than LBs (Sakai, Austin, Witmer, \& Trueb, 1970). The data presented in the present study on LBs are likely to be very relevant to understanding the architecture and formation of other PGB types. It is likely that the aggregation of polyglucosan into networks and B-type crystallization are common features of PGBs. It will be very interesting to compare their CLD profiles and particle size distribution and chemical compositions to understand their architectures and in vitro properties. 
The different levels of polyglucosan association (PGB, network, and particle) are

759

760

761

762

763

764

765

766

767

768

769

770

771

772

773

774

775

776

777

778

779

780

781

782

analogous to the levels of protein aggregation in amyloid disorders. Polyglucosan particles aggregate into networks much like misfolded proteins aggregate into soluble amyloid oligomers. Eventually the polyglucosan compacts into PGBs, and soluble oligomers polymerize into fibrils and plaques, although these higher-orders structures are distinctly different. For example, PGBs contain crystalline $\alpha$-helices, while amyloid contains $\beta$-sheets; polyglucosan fibrils are branched, but amyloid fibrils are not; PGBs are typically not birefringent, in contrast to amyloid. It is possible that for both types of inclusion, the intermediate aggregates are toxic, and the higher-order structures are reactionary, or even protective. There is a significant amount of evidence supporting the toxicity of soluble oligomers rather than amyloid fibrils (Fändrich, 2012; Glabe, 2006). Since LBs are often found in apparently healthy neurons, and degenerating neurons usually lack LBs, some have suggested that the sequestration of polyglucosan into LBs may be a protective mechanism in neuronal cells (Ganesh et al., 2002; Machado-Salas et al., 2012). Corpora amylacea, which appear with aging and various neurological conditions, are also considered a protective response to age-related or pathological degeneration (Rohn, 2015). It has been suggested that the corpora amylacea are a repository for hazardous cellular waste that can be cleared by the immune system (Augé, Duran, Guinovart, Pelegrí, \& Vilaplana, 2018). Both LBs and corpora amylacea are decorated with ubiquitin and p62, a cargo receptor for ubiquitinated proteins that has important roles in autophagy and cellular waste disposal (Augé, Duran, et al., 2018; Criado et al., 2012). Misfolded proteins aggregates are also rich in ubiquitin and p62 (Donaldson et al., 2003). Active cellular sequestration of misfolded proteins has been shown to enhance their response to stress (Escusa-Toret, Vonk, \& Frydman, 2013). Also, conversion of low molecular weight amyloid oligomers to high molecular weight aggregates reduces their toxicity within cells (Cohen, Bieschke, Perciavalle, Kelly, \& Dillin, 2006). It is 
783 possible that when polyglucosan accumulates, cells activate autophagic pathways to sequester

784 the potentially toxic species into compact bodies, analogous to the sequestration of toxic

785 misfolded proteins and amyloid.

786 While PGB formation may be a cellular adaptation mechanism, data from multiple

787 diseases indicate that polyglucosan accumulation is pathogenic. Genetic ablation of glycogen

788 synthase in the brain rescues the neurological phenotype in LD mouse models, and overexpression of glycogen synthase induces polyglucosan accumulation and neurodegeneration in WT flies and mice (Duran, Gruart, Garcia-Rocha, Delgado-Garcia, \& Guinovart, 2014; Duran et al., 2012). Similarly, inhibition of glycogen synthase eliminates

792 PGBs and reverses neurotoxicity in a cell model of Adult Polyglucosan Body Disorder

793 (Kakhlon et al., 2013). Glycogen synthase ablation in WT mice also eliminates the formation of corpora amylacea with aging, and a reduction in glycogen synthesis eliminates polyglucosan deposits and increases lifespan in Drosophila (Sinadinos et al., 2014). Further investigation will be necessary to understand how polyglucosan is sequestered as PGBs in vivo, why various forms of polyglucosan are pathological, and what can be done to alleviate their toxicity.

799

\section{Conclusions}

In this study, we defined the architecture of LBs, the hallmark PGBs of a fatal childhood epilepsy. The hypothesis that LBs contain starch-like properties was confirmed: LBs possess

803 B-type crystallinity like starch, but they lack a lamellar arrangement and contain randomly arrayed helical chains. We show that multiple treatments drastically alter the appearance of

805 LBs, breaking them into particles and/or networks. Like starch, the polyglucosan of LBs

806 undergoes retrogradation, acquiring crystallinity spontaneously over time. We propose a

807 model of LB hierarchical structure and formation based on these results, hypothesizing that in 
vivo, aggregation into networks occurs spontaneously, while sequestration into compact LBs is assisted by proteins. Future work will be required to define how LBs are sequestered, why morphology differs between tissues, and whether the polyglucosan particles, networks or

811 intact PGBs are most toxic. We also present clear nomenclature for describing the various

812 levels of LB structure. These results are also relevant to understanding PGBs found in other

813 diseases and pathologies.

\section{Author Contributions}

$\mathrm{MKB}, \mathrm{AR}$ and $\mathrm{AU}$ purified LBs, performed light microscopy experiments and analyzed data.

817 JLP performed SAXS, WAXS, and TEM experiments and analyzed data. MAS performed

818 SEC and analyzed data. MKB, JLP, MAS and MSG wrote the paper.

819

\section{Conflict of Interest Statement}

821 All authors declare no competing interests.

822

\section{Acknowledgements}

824 This work was supported by the National Institutes of Health [R01 NS070899 to M.S.G., P01 NS097197 to M.S.G., R35 NS116824 to M.S.G., F31 NS093892 to M.K.B], an Epilepsy

826 Foundation New Therapy Commercialization Grant to M.S.G., an award from the Mizutani

827 Foundation for Glycoscience to M.S.G., and the Glyco@ Alps program [ANR-15-IDEX-02].

828 M.K.B. has received funding from the European Union's Horizon 2020 research and innovation programme under the Marie Skłodowska-Curie grant agreement [No. 754510M].

830 M.A.S. is supported by a Mater Research McGuckin Early Career Fellowship, the University 831 of Queensland's Amplify Initiative and Mater Foundation. We acknowledge the NanoBio-

832 ICMG Platform (FR 2607, Grenoble, France) for granting access to the Electron Microscopy 
833 facility and Ziyi Wang for extracting the rat muscle glycogen, which was used as a reference

834 sample for SEC. We also thank Xinle Tan for his technical assistance with SEC experiments,

835 Robert Gilbert for providing access to SEC equipment, Dr. Carole Moncman, Dr. Thomas

836 Wilkop and the UK Light Microscopy Core for technical support, and all members of the

837 Gentry lab and Dr. Craig Vander Kooi for constructive discussions.

838

839 Supplementary Files:

840 Figure S1. Additional confocal images of starch and LBs.

841 Figure S2. Schematic diagram of SAXS and WAXS experimental setup and theory.

842 Figure S3. 2D SAXS and WAXS diffraction patterns.

843 Figure S4. Additional TEM image of heated SmLBs.

844 
Akman, H. O., Oldfors, A., \& DiMauro, S. (2015). Glycogen Storage Diseases of Muscle. In B. T. Darras, H. R. Jones, M. M. Ryan \& D. C. De Vivo (Eds.), Neuromuscular Disorders of Infancy, Childhood, and Adolescence: A Clinician's Approach (pp. 735760): Academic Press

Archibald, A. R., Fleming, I. D., Liddle, A. M., Manners, D. J., Mercer, G. A., \& Wright, A. (1961). 232. $\alpha-1$, 4-Glucosans. Part XI. The absorption spectra of glycogen-and amylopectin-iodine complexes. Journal of the Chemical Society (Resumed), 11831190.

Augé, E., Duran, J., Guinovart, J. J., Pelegrí, C., \& Vilaplana, J. (2018). Exploring the elusive composition of corpora amylacea of human brain. Scientific reports, 8(1), 13525.

Augé, E., Pelegrí, C., Manich, G., Cabezón, I., Guinovart, J. J., Duran, J., \& Vilaplana, J. (2018). Astrocytes and neurons produce distinct types of polyglucosan bodies in Lafora Disease. Glia, in press.

Bahaji, A., Li, J., Ovecka, M., Ezquer, I., Muñoz, F. J., Baroja-Fernández, E., . . Hidalgo, M. (2011). Arabidopsis thaliana mutants lacking ADP-glucose pyrophosphorylase accumulate starch and wild-type ADP-glucose content: further evidence for the occurrence of important sources, other than ADP-glucose pyrophosphorylase, of ADP-glucose linked to leaf starch biosynthesis. Plant and cell physiology, 52(7), 1162-1176.

Berard-Badier, M., Pellissier, J. F., Gambarelli, D., de Barsy, T., Roger, J., \& Toga, M. (1980). The retina in Lafora disease: light and electron microscopy. Albrecht Von Graefes Arch Klin Exp Ophthalmol, 212(3-4), 285-294.

Bertoft, E. (2017). Understanding Starch Structure: Recent Progress. Agronomy, 7(3).

Blazek, J., \& Gilbert, E. P. (2011). Application of small-angle X-ray and neutron scattering techniques to the characterisation of starch structure: A review. Carbohydrate Polymers, 85(2), 281-293.

Brewer, M. K., \& Gentry, M. S. (2019). Brain Glycogen Structure and Its Associated Proteins: Past, Present and Future. Adv Neurobiol, 23, 17-81.

Brewer, M. K., Uittenbogaard, A., Austin, G. L., Segvich, D. M., DePaoli-Roach, A., Roach, P. J., ... Gentry, M. S. (2019). Targeting Pathogenic Lafora Bodies in Lafora Disease Using an Antibody-Enzyme Fusion. Cell Metab.

Buléon, A., Bizot, H., Delage, M. M., \& Pontoire, B. (1987). Comparison of X-ray diffraction patterns and sorption properties of the hydrolyzed starches of potato, wrinkled and smooth pea, broad bean and wheat. Carbohydrate Polymers, 7(6), 461482.

Cafferty, M. S., Lovelace, R. E., Hays, A. P., Servidei, S., Dimauro, S., \& Rowland, L. P. (1991). Polyglucosan body disease. Muscle Nerve, 14(2), 102-107. 
Cai, L., \& Shi, Y.-C. (2013). Self-assembly of short linear chains to A-and B-type starch spherulites and their enzymatic digestibility. Journal of agricultural and food chemistry, 61(45), 10787-10797.

Cameron, R. E., \& Donald, A. M. (1991). Small-angle X-ray scattering and differential scanning calorimetry from starch and retrograded starch. In Food polymers, gels and colloids (pp. 301-309): The Royal Society of Chemistry Cambridge

Cameron, R. E., \& Donald, A. M. (1992). A small-angle X-ray scattering study of the annealing and gelatinization of starch. Polymer, 33(12), 2628-2635.

Cavanagh, J. B. (1999). Corpora-amylacea and the family of polyglucosan diseases. Brain Res Brain Res Rev, 29(2-3), 265-295.

Cleven, R., Van den Berg, C., \& Van Der Plas, L. (1978). Crystal structure of hydrated potato starch. Starch - Stärke, 30(7), 223-228.

Cohen, E., Bieschke, J., Perciavalle, R. M., Kelly, J. W., \& Dillin, A. (2006). Opposing activities protect against age-onset proteotoxicity. Science, 313(5793), 1604-1610.

Criado, O., Aguado, C., Gayarre, J., Duran-Trio, L., Garcia-Cabrero, A. M., Vernia, S., .. . Rodriguez de Cordoba, S. (2012). Lafora bodies and neurological defects in malindeficient mice correlate with impaired autophagy. Hum Mol Genet, 21(7), 1521-1533.

DePaoli-Roach, A. A., Contreras, C. J., Segvich, D. M., Heiss, C., Ishihara, M., Azadi, P., \& Roach, P. J. (2014). Glycogen phosphomonoester distribution in mouse models of the progressive myoclonic epilepsy, Lafora disease. J Biol Chem.

DePaoli-Roach, A. A., Contreras, C. J., Segvich, D. M., Heiss, C., Ishihara, M., Azadi, P., \& Roach, P. J. (2015). Glycogen phosphomonoester distribution in mouse models of the progressive myoclonic epilepsy, Lafora disease. Journal of Biological Chemistry, 290(2), 841-850.

DePaoli-Roach, A. A., Segvich, D. M., Meyer, C. M., Rahimi, Y., Worby, C. A., Gentry, M. S., \& Roach, P. J. (2012). Laforin and malin knockout mice have normal glucose disposal and insulin sensitivity. Hum Mol Genet, 21(7), 1604-1610.

DePaoli-Roach, A. A., Tagliabracci, V. S., Segvich, D. M., Meyer, C. M., Irimia, J. M., \& Roach, P. J. (2010). Genetic depletion of the malin E3 ubiquitin ligase in mice leads to lafora bodies and the accumulation of insoluble laforin. J Biol Chem, 285(33), 25372-25381.

Donald, A. K., Kato, K. L., Perry, P. A., \& Waigh, T. A. (2001). Scattering Studies of the Internal Structure of Starch Granules. Starch, 53(10), 504-512.

Donaldson, K. M., Li, W., Ching, K. A., Batalov, S., Tsai, C.-C., \& Joazeiro, C. A. (2003). Ubiquitin-mediated sequestration of normal cellular proteins into polyglutamine aggregates. Proceedings of the National Academy of Sciences, 100(15), 8892-8897.

Duran, J., Gruart, A., Garcia-Rocha, M., Delgado-Garcia, J. M., \& Guinovart, J. J. (2014). Glycogen accumulation underlies neurodegeneration and autophagy impairment in Lafora disease. Hum Mol Genet, 23(12), 3147-3156. 
Duran, J., \& Guinovart, J. J. (2015). Brain glycogen in health and disease. Mol Aspects Med, 46, 70-77.

Duran, J., Tevy, M. F., Garcia-Rocha, M., Calbo, J., Milan, M., \& Guinovart, J. J. (2012). Deleterious effects of neuronal accumulation of glycogen in flies and mice. EMBO Mol Med, 4(8), 719-729.

Emanuelle, S., Brewer, M. K., Meekins, D. A., \& Gentry, M. S. (2016). Unique carbohydrate binding platforms employed by the glucan phosphatases. Cell Mol Life Sci, 73(14), 2765-2778.

Escusa-Toret, S., Vonk, W. I., \& Frydman, J. (2013). Spatial sequestration of misfolded proteins by a dynamic chaperone pathway enhances cellular fitness during stress. Nature cell biology, 15(10), 1231.

Fändrich, M. (2012). Oligomeric intermediates in amyloid formation: structure determination and mechanisms of toxicity. J Mol Biol, 421(4-5), 427-440.

Ganesh, S., Delgado-Escueta, A. V., Sakamoto, T., Avila, M. R., Machado-Salas, J., Hoshii, Y., ... Yamakawa, K. (2002). Targeted disruption of the Epm2a gene causes formation of Lafora inclusion bodies, neurodegeneration, ataxia, myoclonus epilepsy and impaired behavioral response in mice. Hum Mol Genet, 11(11), 1251-1262.

Gentry, M. S., Afawi, Z., Armstrong, D. D., Delgado-Escueta, A. V., Goldberg, Y. P., Grossman, T. R., . . . Serratosa, J. M. (2020). The 5th International Lafora Epilepsy Workshop: Basic science elucidating therapeutic options and preparing for therapies in the clinic. Epilepsy Behav(In Press).

Gentry, M. S., Dixon, J. E., \& Worby, C. A. (2009). Lafora disease: insights into neurodegeneration from plant metabolism. Trends Biochem Sci, 34(12), 628-639.

Gentry, M. S., Dowen, R. H., 3rd, Worby, C. A., Mattoo, S., Ecker, J. R., \& Dixon, J. E. (2007). The phosphatase laforin crosses evolutionary boundaries and links carbohydrate metabolism to neuronal disease. J Cell Biol, 178(3), 477-488.

Gentry, M. S., Guinovart, J. J., Minassian, B. A., Roach, P. J., \& Serratosa, J. M. (2018). Lafora disease offers a unique window into neuronal glycogen metabolism. $J$ Biol Chem, 293(19), 7117-7125.

Gentry, M. S., Worby, C. A., \& Dixon, J. E. (2005). Insights into Lafora disease: malin is an E3 ubiquitin ligase that ubiquitinates and promotes the degradation of laforin. Proc Natl Acad Sci U S A, 102(24), 8501-8506.

Gerard, C., Planchot, V., Colonna, P., \& Bertoft, E. (2000). Relationship between branching density and crystalline structure of A- and B-type maize mutant starches. Carbohydr Res, 326(2), 130-144.

Gidley, M. J., \& Bulpin, P. V. (1987). Crystallisation of malto-oligosaccharides as models of the crystalline forms of starch: minimum chain-length requirement for the formation of double helices. Carbohydrate Research, 161(2), 291-300. 
Glabe, C. G. (2006). Common mechanisms of amyloid oligomer pathogenesis in degenerative disease. Neurobiology of aging, 27(4), 570-575.

962

963

964

965

966

967

968

969

970

971

972

973

974

975

976

977

978

979

980

981

982

983

984

985

986

987

988

989

990

991

992

993

994

995

996

997

Goldstein, A., Annor, G., Putaux, J.-L., Hebelstrup, K. H., Blennow, A., \& Bertoft, E. (2016). Impact of full range of amylose contents on the architecture of starch granules. International journal of biological macromolecules, 89, 305-318.

Good, C. A. K., H.; Somogyi, M. (1933). The determination of glycogen. J. Biol. Chem., 100, 485-491.

Herrick, M. K., Twiss, J. L., Vladutiu, G. D., Glasscock, G. F., \& Horoupian, D. S. (1994). Concomitant branching enzyme and phosphorylase deficiencies. An unusual glycogenosis with extensive neuronal polyglucosan storage. J Neuropathol Exp Neurol, 53(3), 239-246.

Imberty, A., \& Perez, S. (1988). A revisit to the three - dimensional structure of B - type starch. Biopolymers: Original Research on Biomolecules, 27(8), 1205-1221.

Irimia, J. M., Tagliabracci, V. S., Meyer, C. M., Segvich, D. M., DePaoli-Roach, A. A., \& Roach, P. J. (2015). Muscle glycogen remodeling and glycogen phosphate metabolism following exhaustive exercise of wild type and laforin knockout mice. $J$ Biol Chem.

Ishihara, T., Yokota, T., Yamashita, Y., Takahashi, M., Kawano, H., Uchino, F., .. Y Yamada, M. (1987). Comparative study of the intracytoplasmic inclusions in Lafora disease and type IV glycogenosis by electron microscopy. Acta Pathol Jpn, 37(10), 15911601.

Jacobs, H., Eerlingen, R. C., Rouseu, N., Colonna, P., \& Delcour, J. A. (1998). Acid hydrolysis of native and annealed wheat, potato and pea starches-DSC melting features and chain length distributions of lintnerised starches. Carbohydrate Research, 308(3-4), 359-371.

Jane, J. 1., Chen, Y., Lee, L., McPherson, A., Wong, K., Radosavljevic, M., \& Kasemsuwan, T. (1999). Effects of amylopectin branch chain length and amylose content on the gelatinization and pasting properties of starch. Cereal chemistry, 76(5), 629-637.

Kakhlon, O., Glickstein, H., Feinstein, N., Liu, Y., Baba, O., Terashima, T., . . Lossos, A. (2013). Polyglucosan neurotoxicity caused by glycogen branching enzyme deficiency can be reversed by inhibition of glycogen synthase. J Neurochem, 127(1), 101-113.

Kerly, M. (1930). The solubility of glycogen. Biochemical Journal, 24(1), 67.

Kyle, R. A. (2001). Amyloidosis: a convoluted story. Br J Haematol, 114(3), 529-538.

Lafora, G. R. (1911). Uber des Vorkommen amyloider KJrperchen im innern der Ganglienzellen. Virchows Arch. f. Path. Anat., 205, 295.

Lourdin, D., Putaux, J.-L., Potocki-Véronèse, G., Chevigny, C., Rolland-Sabaté, A., \& Buléon, A. (2015). Crystalline Structure in Starch. In Y. Nakamura (Ed.), Starch: Metabolism and Structure (pp. 61-90). Tokyo: Springer Japan 
Machado-Salas, J., Avila-Costa, M. R., Guevara, P., Guevara, J., Duron, R. M., Bai, D., . . . Delgado-Escueta, A. V. (2012). Ontogeny of Lafora bodies and neurocytoskeleton changes in Laforin-deficient mice. Exp Neurol, 236(1), 131-140.

Majzoobi, M., Seifzadeh, N., Farahnaky, A., \& Mesbahi, G. (2015). Effects of sonication on physical properties of native and cross - linked wheat starches. Journal of Texture Studies, 46(2), 105-112.

Melendez-Hevia, E., Waddell, T. G., \& Shelton, E. D. (1993). Optimization of molecular design in the evolution of metabolism: the glycogen molecule. Biochem J, 295 ( Pt 2), 477-483.

Michen, B., Geers, C., Vanhecke, D., Endes, C., Rothen-Rutishauser, B., Balog, S., \& PetriFink, A. (2015). Avoiding drying-artifacts in transmission electron microscopy: Characterizing the size and colloidal state of nanoparticles. Scientific reports, 5, 9793.

Minassian, B. A. (2001). Lafora's disease: towards a clinical, pathologic, and molecular synthesis. Pediatr Neurol, 25(1), 21-29.

Nakamura, Y. (2015). Starch: Metabolism and Structure. Akita, Japan: Springer.

Nitschke, F., Sullivan, M. A., Wang, P., Zhao, X., Chown, E. E., Perri, A. M., . . Minassian, B. A. (2017). Abnormal glycogen chain length pattern, not hyperphosphorylation, is critical in Lafora disease. 9(7), 906-917.

Nitschke, F., Wang, P., Schmieder, P., Girard, J. M., Awrey, D. E., Wang, T., . . . Minassian, B. A. (2013). Hyperphosphorylation of glucosyl c6 carbons and altered structure of glycogen in the neurodegenerative epilepsy lafora disease. Cell Metab, 17(5), 756767.

Ovecka, M., Bahaji, A., Munoz, F. J., Almagro, G., Ezquer, I., Baroja-Fernandez, E., . . . Pozueta-Romero, J. (2012). A sensitive method for confocal fluorescence microscopic visualization of starch granules in iodine stained samples. Plant Signal Behav, 7(9), 1146-1150.

Perera, A., Meda, V., \& Tyler, R. T. (2010). Resistant starch: A review of analytical protocols for determining resistant starch and of factors affecting the resistant starch content of foods. Food Research International, 43(8), 1959-1974.

Pflüger, E. (1909). Meine Methode der quantitativen Analyse des Glykogenes und die Arteigenthümlichkeit der Substanzen des Thierleibes. Archiv für die gesamte Physiologie des Menschen und der Tiere, 129(6-7), 362-378.

Popov, D., Buléon, A., Burghammer, M., Chanzy, H., Montesanti, N., Putaux, J.-L., . . . Riekel, C. (2009). Crystal structure of A-amylose: A revisit from synchrotron microdiffraction analysis of single crystals. Macromolecules, 42(4), 1167-1174.

Putaux, J.-L., Buleon, A., \& Chanzy, H. (2000). Network formation in dilute amylose and amylopectin studied by TEM. Macromolecules, 33(17), 6416-6422. 
Putaux, J.-L., Molina-Boisseau, S., Momaur, T., \& Dufresne, A. (2003). Platelet nanocrystals resulting from the disruption of waxy maize starch granules by acid hydrolysis. Biomacromolecules, 4(5), 1198-1202.

Putaux, J.-L., Potocki-Véronese, G., Remaud-Simeon, M., \& Buleon, A. (2006). $\alpha$-D-Glucanbased dendritic nanoparticles prepared by in vitro enzymatic chain extension of glycogen. Biomacromolecules, 7(6), 1720-1728.

Qiao, D., Zhang, B., Huang, J., Xie, F., Wang, D. K., Jiang, F., . . Zhu, J. (2017). Hydrationinduced crystalline transformation of starch polymer under ambient conditions. International journal of biological macromolecules, 103, 152-157.

Raben, N., Danon, M., Lu, N., Lee, E., Shliselfeld, L., Skurat, A. V., . . Plotz, P. (2001). Surprises of genetic engineering: A possible model of polyglucosan body disease. Neurology, 56(12), 1739-1745.

Raththagala, M., Brewer, M. K., Parker, M. W., Sherwood, A. R., Wong, B. K., Hsu, S., . . Gentry, M. S. (2015). Structural mechanism of laforin function in glycogen dephosphorylation and lafora disease. Mol Cell, 57(2), 261-272.

Ratnayake, W. S., \& Jackson, D. S. (2006). Gelatinization and solubility of corn starch during heating in excess water: new insights. Journal of Agricultural and Food Chemistry, 54(10), 3712-3716.

Ratnayake, W. S., \& Jackson, D. S. (2009). Starch gelatinization. Adv Food Nutr Res, 55, 221-268.

Reed Jr, G. B., Dixon, J. F., Neustein, J. B., Donnell, G. N., \& Landing, B. H. (1968). Type IV glycogenosis. Patient with absence of a branching enzyme alpha-1, 4-glucan: alpha-1, 4-glucan 6-glycosyl transferase. Laboratory investigation; a journal of technical methods and pathology, 19(5), 546.

Revel, J. P., Napolitano, L., \& Fawcett, D. W. (1960). Identification of glycogen in electron micrographs of thin tissue sections. J Cell Biol, 8(3), 575-589.

Ritte, G., Heydenreich, M., Mahlow, S., Haebel, S., Kotting, O., \& Steup, M. (2006). Phosphorylation of C6- and C3-positions of glucosyl residues in starch is catalysed by distinct dikinases. FEBS Lett, 580(20), 4872-4876.

Roach, P. J. (2002). Glycogen and its Metabolism. Current Molecular Medicine, 2, 101-120.

Roach, P. J., Depaoli-Roach, A. A., Hurley, T. D., \& Tagliabracci, V. S. (2012). Glycogen and its metabolism: some new developments and old themes. Biochem J, 441(3), 763787.

Rohn, T. T. (2015). Corpora Amylacea in Neurodegenerative Diseases: Cause or Effect? Int J Neurol Neurother, 2(3).

Rubio-Villena, C., Viana, R., Bonet, J., Garcia-Gimeno, M. A., Casado, M., Heredia, M., \& Sanz, P. (2018). Astrocytes: new players in progressive myoclonus epilepsy of Lafora type. Hum Mol Genet. 
Rundle, R. E., Foster, J. F., \& Baldwin, R. R. (1944). On the nature of the starch-iodine complex. Journal of the American Chemical Society, 66(12), 2116-2120.

Ryu, J. H., Drain, J., Kim, J. H., McGee, S., Gray-Weale, A., Waddington, L., . . . Stapleton, D. (2009). Comparative structural analyses of purified glycogen particles from rat liver, human skeletal muscle and commercial preparations. Int J Biol Macromol, 45(5), 478-482.

Sakai, M., Austin, J., Witmer, F., \& Trueb, L. (1969). Studies of corpora amylacea. I. Isolation and preliminary characterization by chemical and histochemical techniques. Arch Neurol, 21(5), 526-544.

Sakai, M., Austin, J., Witmer, F., \& Trueb, L. (1970). Studies in myoclonus epilepsy (Lafora body form). II. Polyglucosans in the systemic deposits of myoclonus epilepsy and in corpora amylacea. Neurology, 20(2), 160-176.

Sinadinos, C., Valles-Ortega, J., Boulan, L., Solsona, E., Tevy, M. F., Marquez, M., .. . Guinovart, J. J. (2014). Neuronal glycogen synthesis contributes to physiological aging. Aging Cell, 13(5), 935-945.

Sipe, J. D., \& Cohen, A. S. (2000). Review: history of the amyloid fibril. J Struct Biol, 130(23), 88-98.

Srichuwong, S., Isono, N., Mishima, T., \& Hisamatsu, M. (2005). Structure of lintnerized starch is related to X-ray diffraction pattern and susceptibility to acid and enzyme hydrolysis of starch granules. International journal of biological macromolecules, $37(3), 115-121$.

Stetten, M. R., Katzen, H. M., \& Stetten, D. (1958). A comparison of the glycogens isolated by acid and alkaline procedures. Journal of Biological Chemistry, 232(1), 475-488.

Sullivan, M. A., Nitschke, S., Skwara, E. P., Wang, P., Zhao, X., Pan, X. S., . . Lee, J. P. (2019). Skeletal Muscle Glycogen Chain Length Correlates with Insolubility in Mouse Models of Polyglucosan-Associated Neurodegenerative Diseases. Cell reports, 27(5), 1334-1344. e1336.

Sullivan, M. A., Nitschke, S., Steup, M., Minassian, B. A., \& Nitschke, F. (2017). Pathogenesis of Lafora Disease: Transition of Soluble Glycogen to Insoluble Polyglucosan. Int J Mol Sci, 18(8).

Sullivan, M. A., Powell, P. O., Witt, T., Vilaplana, F., Roura, E., \& Gilbert, R. G. (2014). Improving size-exclusion chromatography separation for glycogen. Journal of Chromatography A, 1332, 21-29.

Sullivan, M. A., Vilaplana, F., Cave, R. A., Stapleton, D., Gray-Weale, A. A., \& Gilbert, R. G. (2010). Nature of $\alpha$ and $\beta$ particles in glycogen using molecular size distributions. Biomacromolecules, 11(4), 1094-1100.

Svegmark, K., Helmersson, K., Nilsson, G., Nilsson, P. O., Andersson, R., \& Svensson, E. (2002). Comparison of potato amylopectin starches and potato starches-influence of year and variety. Carbohydrate Polymers, 47(4), 331-340. 
1112

1113

1114

1115

1116

1117

1118

1119

1120

1121

1122

1123

1124

1125

1126

1127

1128

1129

1130

1131

1132

1133

1134

1135

1136

1137

1138

1139

1140

1141

1142

1143

1144

1145

1146

1147

1148

1149

Swanson, M. A. (1948). Studies on the structure of polysaccharides; relation of the iodine color to the structure. J Biol Chem, 172(2), 825-837.

Tagliabracci, V. S., Girard, J. M., Segvich, D., Meyer, C., Turnbull, J., Zhao, X., . . Roach, P. J. (2008). Abnormal metabolism of glycogen phosphate as a cause for Lafora disease. J Biol Chem, 283(49), 33816-33825.

Tagliabracci, V. S., Heiss, C., Karthik, C., Contreras, C. J., Glushka, J., Ishihara, M., . . Roach, P. J. (2011). Phosphate incorporation during glycogen synthesis and Lafora disease. Cell Metab, 13(3), 274-282.

Tester, R. F., Karkalas, J., \& Qi, X. (2004). Starch-composition, fine structure and architecture. Journal of Cereal Science, 39(2), 151-165.

Thys, R. C. S., Westfahl Jr, H., Noreña, C. P. Z., Marczak, L. D. F., Silveira, N. P., \& Cardoso, M. B. (2008). Effect of the alkaline treatment on the ultrastructure of C-type starch granules. Biomacromolecules, 9(7), 1894-1901.

Tiberia, E., Turnbull, J., Wang, T., Ruggieri, A., Zhao, X. C., Pencea, N., . . Minassian, B. A. (2012). Increased laforin and laforin binding to glycogen underlie Lafora body formation in malin-deficient Lafora disease. J Biol Chem, 287(30), 25650-25659.

Valles-Ortega, J., Duran, J., Garcia-Rocha, M., Bosch, C., Saez, I., Pujadas, L., . . . Guinovart, J. J. (2011). Neurodegeneration and functional impairments associated with glycogen synthase accumulation in a mouse model of Lafora disease. EMBO Mol Med, 3(11), 667-681.

Van Heycop Ten Ham, M. W. (1975). Lafora disease, a form of progressive myoclonus epilepsy. In P. J. Vinken \& G. W. Bruyn (Eds.), Handb Clin Neurol (pp. 382-422). Holland, Amsterdam: North Holland Publishing Company

Virchow, R. (1854). Zur Cellulose-Frage. Virchows Arch., 6(3), 416-426.

Wang, S., Luo, H., Zhang, J., Zhang, Y., He, Z., \& Wang, S. (2014). Alkali-induced changes in functional properties and in vitro digestibility of wheat starch: the role of surface proteins and lipids. Journal of agricultural and food chemistry, 62(16), 3636-3643.

Wikman, J., Blennow, A., \& Bertoft, E. (2013). Effect of amylose deposition on potato tuber starch granule architecture and dynamics as studied by lintnerization. Biopolymers, 99(1), 73-83.

Wikman, J., Blennow, A., Buleon, A., Putaux, J. L., Perez, S., Seetharaman, K., \& Bertoft, E. (2014). Influence of amylopectin structure and degree of phosphorylation on the molecular composition of potato starch lintners. Biopolymers, 101(3), 257-271.

Worby, C. A., Gentry, M. S., \& Dixon, J. E. (2006). Laforin: A dual specificity phosphatase that dephosphorylates complex carbohydrates. J. Biol. Chem., 281(41), 30412-30418.

Yokoi, S., Austin, J., Witmer, F., \& Sakai, M. (1968). Studies in myoclonus epilepsy (Lafora body form). I. Isolation and preliminary characterization of Lafora bodies in two cases. Arch Neurol, 19(1), 15-33. 
1150 Young, L. E., Brizzee, C. O., Macedo, J. K., Murphy, R. D., Contreras, C. J., DePaoli-Roach, A. A., ... Sun, R. C. (2019). Accurate and sensitive quantitation of glucose and glucose phosphates derived from storage carbohydrates by mass spectrometry. 